Indiana University Mathematics Journal (c)

Article electronically published on February 17th, 2009.

\title{
Nonlinear Stability of High Lewis Number Combustion Fronts
}

\author{
ANNA GHAZARYAN
}

\begin{abstract}
In this paper a mathematical model is considered that describes combustion processes characterized by a very high Lewis number. The model is known to support a wavefront that asymptotically connects the completely burned and the unburned states, and that is unique up to translation. The stability of the front has not yet been investigated beyond the spectral level. The essential spectrum of the linearization of the system about the front touches to the imaginary axis, therefore, even in a parameter regime that guarantees absence of the unstable discrete spectrum, spectral information is not definitive. There exists an exponentially weighted norm that stabilizes the front on the linear level. The nonlinear stability in that exponentially weighted norm cannot be simply inferred from the spectral stability because the nonlinearity is not smooth in that norm. We use the interplay of the norms with and without exponential weight to overcome this issue, and show that the front in the comoving frame is nonlinearly stable in the exponentially weighted norm with respect to a special class of perturbations.
\end{abstract}

\section{INTRODUCTION}

In this paper we study the nonlinear stability of a traveling wavefront that occurs in a model for high Lewis number combustion. Before describing the model in detail, we will discuss its key features on the example of a general reaction-diffusion system. Assume that a nonlinear parabolic equation

$$
\partial_{t} U=D \partial_{x x} U+N(U), \quad U \in \mathbb{R}^{n}, x \in \mathbb{R}, t \geq 0,
$$


has a front solution $H(\xi)=H(x-c t)$. Here $D=\left(d_{1}, \ldots, d_{n}\right), d_{i}>0$ for $i=1, \ldots n$, is the diffusion matrix, and $N(U)$ is a smooth, nonlinear function of $U$. The first step in the stability analysis of the front involves locating the spectrum of the linearization of the system (1.1) about the front $H$,

$$
\partial_{t} U=D \partial_{\xi \xi} U+c \partial_{\xi} U+\partial_{U} N(H) U
$$

The spectrum of the linearization contains critical information about the nonlinear stability of the wave. We next assume that the wave is spectrally stable, i.e. there are no points of the spectrum with non-negative real parts, except at the origin. The two possible options for the spectrum are illustrated in Fig. 1.1. The parabolic shape of the essential spectrum is due to the presence of diffusive terms. The eigenvalue at the origin, that may be isolated or embedded in the essential spectrum, is due to the translational invariance of the system. If the continuous spectrum is bounded away from the imaginary axis (Fig. 1.1(b)) and the eigenvalue at the origin is simple, then the wave is nonlinearly stable [14].

The case of marginal stability (Fig. 1.1(b)), which is characterized by the essential spectrum touching the imaginary axis at the origin, at a quadratic tangency, is one of the most interesting and nontrivial situations that may occur in the stability analysis of traveling waves. In this case, the information from the spectrum is not definitive. In other words, nonlinear stability does not simply follow from the spectral information. On the linear level, one expects that perturbations to the front will decay but not exponentially. There are techniques for dealing with marginally stable fronts that involve exponential or algebraic weights. A serious issue with using these weights is that the nonlinearity does not always behave nicely in the weighted spaces that stabilize the linear problem. For example, in the case of this combustion model, the nonlinearity is not differentiable in the weighted space that is needed for linear stability.

To overcome the non-differentiability issue, we take a different approach. The equation for the perturbation $\tilde{U}$ to the front and the equation for the weighted perturbation $\tilde{W}=\rho \tilde{U}$, where $\rho$ is an appropriate weight, will not be considered separately of each other. Instead we will build a hybrid system: a nonlinear equation for $Z=(\tilde{U}, \tilde{W})$, where the coupling occurs on both the linear and nonlinear levels in such a way that we can achieve two goals. First, we want the nonlinearity, as a function of $Z$, to be sufficiently smooth. Second, using the structure of the front, we will write the linear terms in a convenient form that will allow us to show that the linear terms dominate the nonlinearity. We can show in this case that marginal stability implies nonlinear stability in the weighted norm. Moreover, we show that even in the norm without weight, if initial perturbations are sufficiently localized, the perturbed solution will converge to a translate of the front, although not exponentially fast. More details about this approach are provided in the next few sections. 

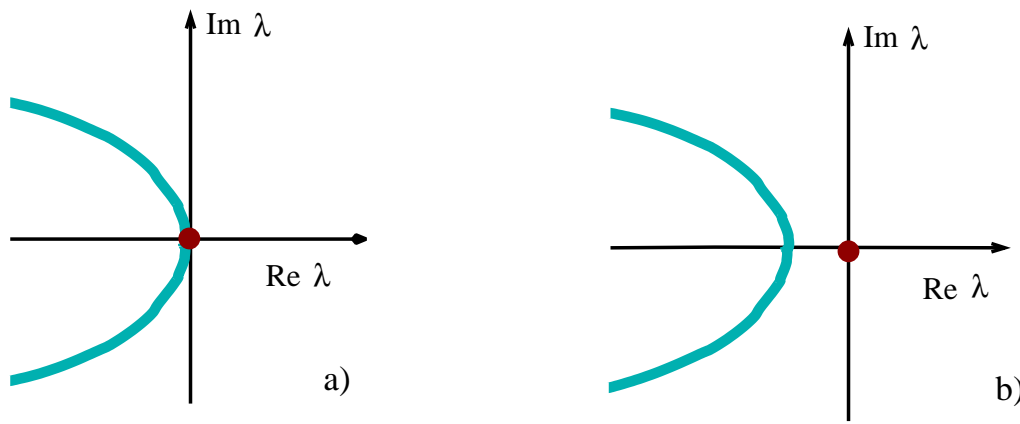

FIGURE 1.1. The spectrum of the linearization about the wave is located on and to the left of the parabola: a) marginally stable case, b) linearly stable case. The eigenvalue at the origin is due to translational invariance of the system. In this schematic picture stable discrete eigenvalues are disregarded.

1.1. Model. The following model is known to describe propagation of combustion waves in the case of premixed fuel, with no heat loss, in one spatial dimension $x \in \mathbb{R}$,

$$
\begin{aligned}
& \partial_{t} u_{1}=\partial_{x x} u_{1}+u_{2} \Omega\left(u_{1}\right), \\
& \partial_{t} u_{2}=\varepsilon \partial_{x x} u_{2}-\beta u_{2} \Omega\left(u_{1}\right) .
\end{aligned}
$$

Here $u_{1}$ is the temperature and $u_{2}$ is the concentration of the fuel. The reaction rate has the form of an Arrhenius law without ignition cutoff: $\Omega\left(u_{1}\right)=e^{-1 / u_{1}}$ for $u_{1}>0$ and $\Omega\left(u_{1}\right)=0$ otherwise. The system has two parameters. One is the exothermicity $\beta>0$ which is the ratio of the activation energy to the heat of the reaction. The other is the reciprocal of the Lewis number $\varepsilon=1 / \mathrm{Le}>0$. Therefore, $\varepsilon$ represents the ratio of the fuel diffusivity to the heat diffusivity. When $0<\varepsilon \ll 1$, i.e., Le is very large but finite, system (1.3) describes burning of very high density fluids at high temperatures [2]. It also can be used as a model for combustion of solid fuels $(\mathrm{Le}=\infty)$ when melting occurs in the reaction zone. The system in a liquid phase then is characterized by a finite, but large, Lewis number.

1.2. Existence of the front. The combustion fronts that asymptotically connect

$$
\left(u_{1}, u_{2}\right)=(1 / \beta, 0) \text { at }-\infty \text { and }\left(u_{1}, u_{2}\right)=(0,1) \text { at } \infty \text {, }
$$

are in the center of this study. The boundary conditions represent the physical state where the fuel is completely burned, i.e., $u_{2}=0$, and the maximal temperature $u_{1}=1 / \beta$ is reached, and the state when none of the fuel is yet burned 
and the temperature $u_{1}$ is still zero. The direction of propagation is chosen so the front moves leaving the burned state behind. As a solution preserving its shape, a front is a function of a single variable $\xi=x-c t$, where $c$ represents the velocity of the front. In the co-moving coordinate frame, (1.3) reads

$$
\begin{aligned}
& \partial_{t} u_{1}=\partial_{\xi \xi} u_{1}+c \partial_{\xi} u_{1}+u_{2} \Omega\left(u_{1}\right), \\
& \partial_{t} u_{2}=\varepsilon \partial_{\xi \xi} u_{2}+c \partial_{\xi} u_{2}-\beta u_{2} \Omega\left(u_{1}\right),
\end{aligned}
$$

where now $u_{1}$ and $u_{2}$ are functions of $\xi$ and $t$. A front is sought as a stationary solution of (1.5),

$$
\begin{aligned}
u_{1}^{\prime \prime}+c u_{1}^{\prime} & =-u_{2} \Omega\left(u_{1}\right), \\
\varepsilon u_{2}^{\prime \prime}+c u_{2}^{\prime} & =\beta u_{2} \Omega\left(u_{1}\right),
\end{aligned}
$$

where the derivative is with respect to $\xi$. Equation (1.6) is called the traveling wave equation.

For (1.6) there are solutions that decay to the rest state at $\infty$ at algebraic rates, but these are considered to be of little interest [26]: one of the reasons for such discrimination is that any amount of heat loss in the physical system will destroy these algebraicly decaying solutions.

It is known that a unique $c>0$ exists for which system (1.6) has a nontrivial solution that asymptotically connects the rest states (1.4): in an appropriate scaling, the traveling wave equation (1.6) with $0<\varepsilon<1$ falls in a class of equations described in [3], where Leray-Schauder degree theory has been used to prove the existence and uniqueness of the front. For the same system as in [3] the existence and uniqueness questions have been addressed in [17] from the point of view of phase plane analysis.

For $\varepsilon$ sufficiently small, an essentially different approach to the proof of the existence and uniqueness of the front is based on geometric singular perturbation theory [16]. System (1.6) has a slow-fast structure. In the limiting case of $\varepsilon=0$ the flow is restricted to a two-dimensional invariant manifold. The manifold is normally hyperbolic and attracting; therefore, by Fenichel's First theorem [5], it perturbs to an attracting manifold invariant for the flow with sufficiently small $\varepsilon>0$. For the reduced problem, the lower dimension of the problem can be used to show that the front in the $\varepsilon=0$ case is formed as a transversal intersection of relevant invariant manifolds. Transversality can be proved by Melnikov integral calculation [2], or by following the blueprint of the proof of the existence and uniqueness of subsonic detonation waves [8].

The slow-fast structure of the wave can be used not only to prove the existence and uniqueness of the front for the perturbed system, but also to show (see [9]) that the spectrum of the front with sufficiently small $\varepsilon>0$ is a perturbation of order $\varepsilon$ of the spectrum of the front with $\varepsilon=0$. In contrast to spectral information, further stability analysis of the front for $\varepsilon=0$ and $0<\varepsilon \ll 1$ calls 
for essentially different approaches due to significantly different properties of the linearized operators. The case of nonzero $\varepsilon$ is discussed in this paper. The case of $\varepsilon=0$ is studied in [10]. The analysis in [10] is independent of the results for $\varepsilon>0$, although it is possible that the relation between the stability of fronts in the cases of zero and nonzero $\varepsilon$ may be revealed in a way similar to one used in [30] or [28].

This approach is particularly useful for our purposes because the geometric construction of the front provides an additional piece of information: the unique front of (1.3) converges to the rest states (1.4) exponentially fast. This fact is crucial for the proof of the nonlinear stability result in the present paper.

1.3. Stability. The unique front of (1.3) is denoted by $H=\left(h_{1}, h_{2}\right)$, and its corresponding speed by $c$. The linearization of (1.5) about $H$ is given by

$$
\begin{aligned}
& \partial_{t} p_{1}=\partial_{\xi \xi} p_{1}+c \partial_{\xi} p_{1}+\Omega\left(h_{1}\right) p_{2}+h_{2} \Omega_{h_{1}}\left(h_{1}\right) p_{1}, \\
& \partial_{t} p_{2}=\varepsilon \partial_{\xi \xi} p_{2}+c \partial_{\xi} p_{2}-\beta \Omega\left(h_{1}\right) p_{2}-\beta h_{2} \Omega_{h_{1}}\left(h_{1}\right) p_{1},
\end{aligned}
$$

where $\Omega_{h_{1}}\left(h_{1}\right)=\Omega\left(h_{1}\right) / h_{1}^{2}$. The eigenvalue problem reads

$$
\begin{aligned}
& \lambda p_{1}=\partial_{\xi \xi} p_{1}+c \partial_{\xi} p_{1}+\Omega\left(h_{1}\right) p_{2}+h_{2} \Omega_{h_{1}}\left(h_{1}\right) p_{1}, \\
& \lambda p_{2}=\varepsilon \partial_{\xi \xi} p_{2}+c \partial_{\xi} p_{2}-\beta \Omega\left(h_{1}\right) p_{2}-\beta h_{2} \Omega_{h_{1}}\left(h_{1}\right) p_{1} .
\end{aligned}
$$

A traveling wave is called spectrally stable if the spectrum of the linearization of the system about the traveling wave is contained in the left half-plane $\{\lambda \in$ $\mathbb{C}: \operatorname{Re} \lambda \leq 0\}$. Generally speaking, the spectral stability need not imply the linear stability of the traveling wave, i.e., the decay of the solutions of the linearized PDE (1.7).

If the linearized operator is sectorial, the linear stability is guaranteed if there exists $B>0$ such that the spectrum belongs to the half-plane $\{\lambda \in \mathbb{C}: \operatorname{Re} \lambda<-B\}$ with the exception of a simple eigenvalue at zero that is caused by translation symmetry. For (1.8) with sufficiently small $\varepsilon>0$ it has been shown in [9] using the Stability Index technique [1] that the translational eigenvalue $\lambda=0$ is simple. The proof is based on the reduction of the spectral stability analysis of the case $0<\varepsilon \ll$ 1 to the stability analysis of the case $\varepsilon=0$. For $\varepsilon=0$ the multiplicity of the zero eigenvalue is calculated in [10] using the Evans function. Numerical investigations $[2,13,25]$, for both $\varepsilon=0$ and $\varepsilon>0$, also confirm that the translational eigenvalue is simple.

There is numerical evidence [13] that the spectrum of the front $(0<\varepsilon \ll 1)$ strongly depends on the parameter $\beta$ : there exist $\beta_{0}=O(1)$ such that for $\beta<\beta_{0}$ the linearization about the front does not possess any unstable eigenvalues. If $\beta$ increases a pair of complex conjugate eigenvalues crosses the imaginary axis from left to right, causing a so-called pulsating instability. The occurrence of a "Hopf" bifurcation, with the speed of the front as the bifurcation parameter, has also been 


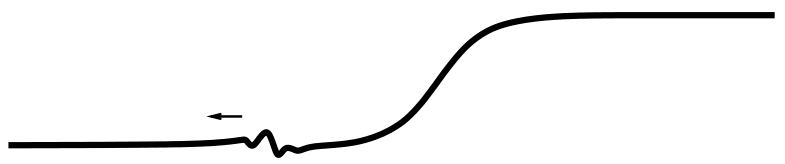

FIGURE 1.2. In the frame moving with the front, a small, sufficiently localized perturbation is transported to $-\infty$.

discussed in [25]. We avoid calling this bifurcation a Hopf bifurcation in a regular sense because the spectral picture is not that of a generic case of a Hopf bifurcation: in addition to a pair of isolated eigenvalues crossing the imaginary axis, the essential spectrum is touching the imaginary axis for all $\beta$. Theoretically, the occurrence of a Hopf bifurcation is not clear at all, or guaranteed. The LyapunovSchmidt method and the center manifold theorem are not applicable to analyze the bifurcation scenario. An example of the analysis of the nature of the bifurcation in a situation with similar spectral characteristics can be found in [4]. There, for a reaction-diffusion-convection system, the trivial solution and the bifurcating time-periodic patterns are proved to be nonlinearly stable against spatially localized perturbations.

The case of $\beta$ that yields eigenvalues on the imaginary axis or to the right of it is out of scope of this paper. In what follows a parameter regime is assumed when there are no eigenvalues with non-negative real parts other than that at the origin.

In Section 2.1 the location of the essential spectrum of the linearization of (1.3) about the front is studied and it is shown that the essential spectrum touches the imaginary axis at the origin. The boundaries of the essential spectrum are found using the linear dispersion relations at asymptotic states at $\pm \infty: d^{ \pm}(\lambda, \nu)=$ 0 that are relations between temporal eigenvalues $\lambda$ and spatial eigenvalues $v$ at the asymptotic rest states. The group velocity $c_{g r}=-(\mathrm{d} \operatorname{Im} \lambda) /(\mathrm{d} v)$ is calculated. The sign of the group velocity, $c_{g r}<0$, implies that the system on the linear level transports perturbations in the direction opposite to the propagation of the front, to $-\infty$ (see Fig.1.2). Perturbations are stabilized in a norm with exponential weights with positive rates. The essential spectrum can be shifted to the open left half-plane using exponential weights with positive rates (Section 2.2).

For fixed $\varepsilon>0$ the linearized operator is sectorial $[14,20]$ as a perturbation of the Laplacian by lower-order derivatives and bounded operators. The linear stability of the front in appropriate exponentially weighted spaces follows from the spectral stability. This is not the case for the system with $\varepsilon=0$, where the spectrum of the linearized operator contains a vertical line (the imaginary axis) and therefore the linearized operator is not sectorial; see [10] for details.

The next question is whether spectral stability implies stability of the solution to the full PDE (1.3), in other words, nonlinear stability. Two scenarios are referred to as nonlinearly stable: solutions initially close to the wave can be attracted to the traveling wave itself or some fixed translate of it (orbital stability, or nonlinear stability with asymptotic phase) or they can stay in a small neighborhood of 
the set of all translates of the front, but never settle down to a particular one. For the model considered here, in the co-moving frame, the front will be proved to be orbitally stable in a weighted norm with respect to small perturbations from some exponentially weighted space.

The nonlinear terms in our problem have a specific structure: in exponentially weighted norms the nonlinearity is well defined and uniformly bounded, but its derivative is not. Indeed, the nonlinearity in the equation for perturbation $V=$ $\left(v_{1}, v_{2}\right)$ to the front, when transformed to the weighted variable $W=\left(w_{1}, w_{2}\right)=$ $e^{\alpha \xi} V$, will contain terms such as $w_{2} \exp \left(-e^{\alpha \xi} / w_{1}\right)$, where the partial derivative with respect to $w_{1}$ is not a bounded function. Therefore the nonlinear stability of the front $H$ in weighted spaces cannot be inferred from linear stability. Instead, we use an approach that is based on the interaction of a spatially uniform norm and the exponentially weighted norm. This technique originated in [21] in the Hamiltonian context. If one can obtain a priori estimates for the perturbation to the front in the space without weight, for instance in the space $C^{1}$ of functions uniformly bounded together with their derivatives, and show that the perturbation stays bounded and sufficiently small, then the nonlinear terms in the equation for weighted perturbations can be controlled as linear in $W$. For example,

$$
\left\|e^{\alpha \xi} v_{2} \exp \left(-\frac{1}{v_{1}}\right)\right\|_{H^{1}} \leq M\left\|\exp \left(-\frac{1}{v_{1}}\right)\right\|_{C^{1}}\left\|w_{2}\right\|_{H^{1}}, \quad M>0 .
$$

This technique has been used to prove the nonlinear stability of the essentially unstable fronts characterized by small-amplitude Turing patterns appearing in the neighborhood of the rest state triggering the instability [7,11]. Another example of a successful application of this technique has been given in [4] where a reactiondiffusion-convection system is considered that has the essential spectrum up to the imaginary axis for all values of the bifurcation parameter, while a pair of imaginary eigenvalues crosses the imaginary axis at the bifurcation point.

In this paper, in addition to a nonlinear stability result in weighted spaces, we show that the interaction of the norms can also be useful to find rates of convergence of the perturbation of the front of (1.3) in a norm without a weight.

We will study the stability of the front against perturbations that, in addition to being from $H^{1}$, decay on $+\infty$ faster than $e^{-\alpha \xi}$ for some $\alpha>0$. In other words, perturbations are restricted to the intersection of $H^{1}$ and

$$
H_{\alpha}^{1}=\left\{f: \int\left(f(s)^{2}+f^{\prime}(s)^{2}\right) e^{2 \alpha s} \mathrm{~d} s<\infty\right\},
$$

an exponentially weighted $H^{1}$. This restriction, although it appears to be an analytic convenience, does not contradict the natural properties of the application. Indeed, the perturbations related to variations in temperature, "hot spots," or inhomogeneities of the fuel can be assumed to be spatially localized. Moreover, in the neighborhood of the completely unburned state, at $+\infty$, if initial perturbations to the front are allowed that converge at $+\infty$ very slowly, then it is possible 
that the perturbed front will converge to one of the non-physical fronts that decay to the unburned state at algebraic rates. To prevent this from happening, one controls the rate of convergence at $+\infty$ by choosing an appropriate $\alpha$. Therefore considering perturbations from $H^{1} \cap H_{\alpha}^{1}$ seems to be a natural choice.

It will be shown below that, for some carefully chosen $\alpha>0$, in the co-moving frame the front is nonlinearly stable with asymptotic phase with respect to such perturbations. More precisely, the following theorem holds.

Theorem 1.3.1. Assume that parameter $\beta$ is such that the front $H$ is spectrally stable. There exist positive constants $\alpha, \rho^{*}$, and $\nu^{*}$ such that the following is true: For every $0<\rho \leq \rho^{*}$, there exists $K>0$ such that for $V^{0} \in H^{1} \cap H_{\alpha}^{1}$ with

$$
\left\|V^{0}\right\|_{H^{1}}+\left\|V^{0}\right\|_{H_{\alpha}^{1}} \leq \rho,
$$

equation (1.5) with initial data $U_{0}=H+V_{0}$ has a unique global solution $U(t)$, which can be expressed as

$$
U(x, t)=H(x-c t-q(t))+V(x, t)
$$

for an appropriate real-valued function $q(t)$, and there is a $q_{*} \in \mathbb{R}$ so that

$$
|q(t)| \leq K \rho, \quad\|V(t)\|_{H_{\alpha}^{1}}+\left|q(t)-q_{*}\right| \leq K \rho \mathrm{e}^{-v^{*} t}
$$

for $t \geq 0$.

Moreover, there exists a constant $\hat{K}>0$ such that for $t \geq 0$ the following is true for $V=\left(v_{1}, v_{2}\right)$ :

$$
\begin{aligned}
\left\|v_{2}(t)\right\|_{H_{1}} & \leq \hat{K} \rho \mathrm{e}^{-\left(v^{*} / 2\right) t}, \\
\left\|v_{1}(t)\right\|_{H^{1}} & \leq \hat{K} \rho,
\end{aligned}
$$

i.e., $v_{2}$ converges to 0 exponentially and $v_{1}$ is bounded.

If, in addition to (1.9), $V^{0}$ is in $L^{1}$ space, then $v_{1}$ decays algebraically fast in $L^{\infty}$-norm: there exists $\tilde{K}>0$ such that

$$
\left\|v_{1}(t)\right\|_{L^{\infty}} \leq \tilde{K} \frac{1}{t^{1 / 2}}, \quad t>0
$$

The theorem says that given that initial perturbations are sufficiently small in the regular $H^{1}$ norm and the weighted $H_{\alpha}^{1}$ norm, how far away the perturbed solution will slide before settling at a translate of the front is determined by how small the initial perturbation is. The reaction for such class of perturbations will not stop until all of the fuel is burned. The behavior of the temperature is in agreement with the usual expectations of heat diffusion: the perturbation decays like a heat kernel. Because of the weight, we know that initial conditions are exponentially localized at $+\infty$. The requirement on $L^{1}$-norm of the initial perturbation 
can be interpreted as an additional restriction on how fast the perturbation should decay at $-\infty$ for the front to be stable.

At the end of the introduction we want to mention just a few papers where nonlinear results for marginally stable fronts have been obtained (see also references therein and a review paper [23] for additional relevant literature).

A well-known example of a stability result obtained using exponential weights for fronts in scalar equations is presented in [24]. There an assumption is made that the wave itself belongs to the weighted space and the inverse of the weight is bounded. Algebraic weights have been used in [18] under the assumption that the nonlinearity is defined in the weighted space. In conservation laws, the nonlinear stability of marginally stable viscous shocks has been analyzed in $[15,18,27,29]$ using sharp pointwise estimates on the Green's function of the linearization. Instability criteria for shock waves has been formulated in [6] using a construction of the Evans function that is valid in the region containing the intersection of the essential spectrum with the imaginary axis.

The techniques used for viscous shock have been generalized to reduce the question of the nonlinear stability to the spectral information for traveling wave solutions of an abstract viscous combustion model including both Majda's model and the full reacting compressible Navier-Stokes equations with artificial viscosity [19]. The spectrum is found by means of the Evans function.

An interesting aspect of nonlinear stability of fronts in viscous conservation laws is that the weight can be chosen independently of viscosity if the viscosity is sufficiently small. The stability of the corresponding shocks in hyperbolic (zero viscosity) conservation laws in certain exponentially weighted spaces then is a necessary condition for the stability of viscous shocks [30]. The same mathematical phenomenon takes place for balance laws [28].

The key feature of the stability analysis for the above mentioned papers is that the nonlinear stability follows from the information obtained from the spectral analysis of either the system with or without the weight as the effect of the corresponding nonlinearity is assumed or shown to be negligible compared to the linear terms. As we already said, this is not the case for our model. The proof is based on the interaction of the variables with and without the weight.

The plan of the paper is as follows. In Section 2 we discuss the location of the spectrum of the front, the effects of exponential weights on the spectrum, and the stability of the front in the weighted space on the linear level. The nonlinear stability is addressed in Section 3. The proof of Theorem 1.3.1 consists of two parts. First, using weighted spaces, we show that the front is nonlinearly stable provided the perturbations to the front are sufficiently small in the norm without weight. Afterwards, we establish a priori estimates that show that the norm of the perturbations can be controlled in terms of the norm of the initial data. We conclude by analyzing an interesting case when the initial data satisfy some additional assumptions. 


\section{SPECTRAL STABILITY}

We denote the linear operator defined by the right hand side of (1.6) by

$$
\begin{aligned}
\mathcal{M}_{0} P=\mathcal{M}\left(\partial_{\xi}\right) P & =\left(\begin{array}{cc}
\partial \xi \xi+c \partial_{\xi}+h_{2} \Omega_{h_{1}}\left(h_{1}\right) & \Omega\left(h_{1}\right) \\
-\beta h_{2} \Omega_{h_{1}}\left(h_{1}\right) & \varepsilon \partial \xi \xi+c \partial_{\xi}-\beta \Omega\left(h_{1}\right)
\end{array}\right) P, \\
P & =\left(\begin{array}{l}
p_{1} \\
p_{2}
\end{array}\right) .
\end{aligned}
$$

The linearized operator restricted to the exponentially weighted space $H_{\alpha}^{1}$ is then given by $\mathcal{M}_{\alpha}=\mathcal{M}\left(\partial_{\xi}-\alpha\right)$. The essential spectrum is the complement of the point spectrum in the spectrum of the operator. First, the location of the essential spectrum for $\mathcal{M}_{0}$, and, second, for $\mathcal{M}_{\alpha}$, is found in the next two subsections. In what follows $\varepsilon$ is a small but fixed positive number.

2.1. Essential spectrum. The eigenvalue problem (1.8), written as a first order ODE, reads

$$
\begin{aligned}
& p_{1}^{\prime}=q_{1}, \\
& q_{1}^{\prime}=-c q_{1}-\Omega\left(h_{1}\right) p_{2}+\left(\lambda-h_{2} \Omega_{h_{1}}\left(h_{1}\right)\right) p_{1}, \\
& p_{2}^{\prime}=q_{2}, \\
& q_{2}^{\prime}=\frac{1}{\varepsilon}\left(-c q_{2}+\beta \Omega\left(h_{1}\right) p_{2}+\beta h_{2} \Omega_{h_{1}}\left(h_{1}\right) p_{1}+\lambda p_{2}\right) .
\end{aligned}
$$

The right-hand side of this system is an action of the matrix

$$
M(\xi, \lambda)=\left(\begin{array}{cccc}
0 & 1 & 0 & 0 \\
\lambda-h_{2} \Omega_{h_{1}}\left(h_{1}\right) & -c & -\Omega\left(h_{1}\right) & 0 \\
0 & 0 & 0 & 1 \\
\frac{\beta}{\varepsilon} h_{2} \Omega_{h_{1}}\left(h_{1}\right) & 0 & \frac{1}{\varepsilon}\left(\lambda+\beta \Omega\left(h_{1}\right)\right) & -\frac{c}{\varepsilon}
\end{array}\right)
$$

on the vector $\left(p_{1}, q_{1}, p_{2}, q_{2}\right)^{T}$. Let $M^{ \pm}(\lambda)=\lim _{\xi \rightarrow \pm \infty} M(\xi, \lambda)$. Then

$$
M^{-}(\lambda, \varepsilon)=\left(\begin{array}{cccc}
0 & 1 & 0 & 0 \\
\lambda & -c & -e^{-\beta} & 0 \\
0 & 0 & 0 & 1 \\
0 & 0 & \frac{1}{\varepsilon}\left(\lambda+\beta e^{-\beta}\right) & -\frac{c}{\varepsilon}
\end{array}\right) \text { and } M^{+}(\lambda)=\left(\begin{array}{cccc}
0 & 1 & 0 & 0 \\
\lambda & -c & 0 & 0 \\
0 & 0 & 0 & 1 \\
0 & 0 & \frac{\lambda}{\varepsilon} & -\frac{c}{\varepsilon}
\end{array}\right) \text {. }
$$


The eigenvalues of $M^{ \pm}(\lambda)$ are called the spatial eigenvalues as opposed to the temporal eigenvalues $\lambda$. The eigenvalues of $M^{-}(\lambda)$ are

$$
\begin{array}{ll}
\kappa_{1}^{-}=\frac{1}{2 \varepsilon}\left(-c-\sqrt{c^{2}+4\left(\lambda+\beta e^{-\beta}\right)}\right), & \kappa_{3}^{-}=\frac{1}{2}\left(-c-\sqrt{c^{2}+4 \lambda}\right), \\
\kappa_{2}^{-}=\frac{1}{2 \varepsilon}\left(-c+\sqrt{c^{2}+4\left(\lambda+\beta e^{-\beta}\right)}\right), & \kappa_{4}^{-}=\frac{1}{2}\left(-c+\sqrt{c^{2}+4 \lambda}\right) .
\end{array}
$$

When $\lambda$ crosses the imaginary axis from right to left, $\kappa_{4}^{-}$crosses the imaginary axis from right to left. The boundaries of the essential spectrum due to the behavior at $-\infty$ are curves

$$
\left\{\lambda=-\varepsilon v^{2}+\operatorname{civ}-\beta e^{-\beta} ; \nu \in \mathbb{R}\right\} \cup\left\{\lambda=-v^{2}+\operatorname{civ} ; \nu \in \mathbb{R}\right\} .
$$

The eigenvalues of $M^{+}(\lambda)$ are

$$
\begin{array}{ll}
\kappa_{1}^{+}=\frac{1}{2 \varepsilon}\left(-c-\sqrt{c^{2}+4 \lambda}\right), & \kappa_{3}^{+}=\frac{1}{2}\left(-c-\sqrt{c^{2}+4 \lambda}\right), \\
\kappa_{2}^{+}=\frac{1}{2 \varepsilon}\left(-c+\sqrt{c^{2}+4 \lambda}\right), & \kappa_{4}^{+}=\frac{1}{2}\left(-c+\sqrt{c^{2}+4 \lambda}\right) .
\end{array}
$$

When $\lambda$ crosses the imaginary axis from right to left, both eigenvalues with positive real parts, $\kappa_{2}^{-}, \kappa_{4}^{-}$, cross the imaginary axis from right to left, and coincide at $\lambda=0$. The boundaries of the essential spectrum due to the behavior at $+\infty$ are given by the curves

$$
\left\{\lambda=-\varepsilon \nu^{2}+\operatorname{civ} ; \nu \in \mathbb{R}\right\} \cup\left\{\lambda=-\nu^{2}+\operatorname{civ} ; \nu \in \mathbb{R}\right\} .
$$

The set of curves (2.2)-(2.3) divides the complex plane into regions that are either covered by spectrum or, otherwise, contain only discrete eigenvalues. There is a component which contains the right half-plane of the complex plane. From [9] it is known that there can be no eigenvalues with very large positive real parts. Therefore the region to the right of the rightmost parabola from (2.2) and (2.3)

$$
\left\{\lambda=-\varepsilon v^{2}+\operatorname{civ} ; \nu \in \mathbb{R}\right\}
$$

contains only the discrete spectrum, i.e., isolated eigenvalues of finite multiplicity. The essential spectrum is bounded on the right by (2.4) and includes that curve (see Fig. 2.2).

The direction in which spatial eigenvalues cross the imaginary axis is related to the group velocity and determines where, on the linear level, the system transports initial perturbations. In this case the system convects toward $-\infty$. Indeed, this follows from the dynamics of the spatial eigenvalues (see Fig. 2.1 for a schematic description). 

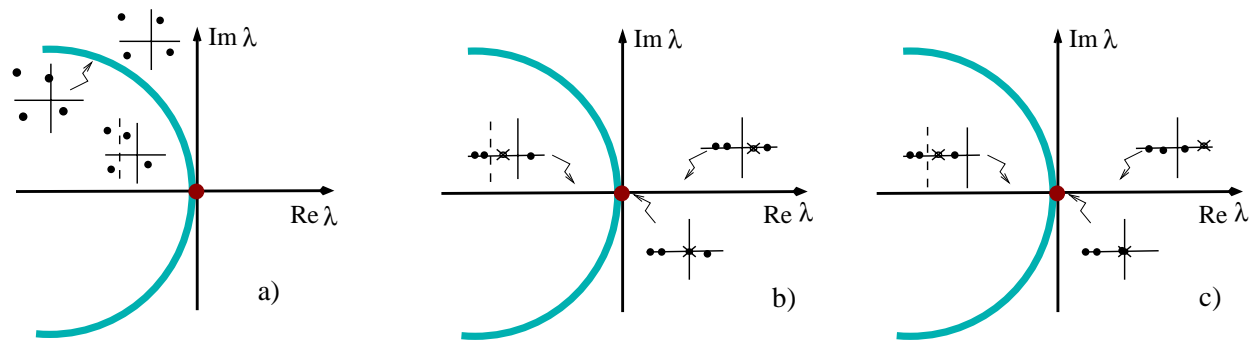

FIGURE 2.1. The picture shows the location of the spatial eigenvalues depending on the location of $\lambda$ relative to the rightmost boundary of the essential spectrum: a) $\operatorname{Im} \lambda \neq 0$, for either $M^{-}$or $M^{+}$; b) $\operatorname{Im} \lambda=0$ for $M^{-}$; c) $\operatorname{Im} \lambda=0$ for $M^{+}$. The dotted line shows that spatial eigenvalues that cross the imaginary axis are still separated from the eigenvalues with strongly negative real parts by a vertical line $\operatorname{Re} \lambda=-d<0$. This makes it possible to chose an exponential weight.

At $\pm \infty$, when $\lambda$ is to the right of the essential spectrum, we have $\operatorname{Re} \kappa_{2}^{ \pm} \geq$ $\operatorname{Re} \kappa_{4}^{ \pm}>0>\operatorname{Re} \kappa_{3}^{ \pm} \geq \operatorname{Re} \kappa_{1}^{ \pm}$. When $\lambda$ crosses the essential spectrum at least one of the spatial eigenvalues crosses the imaginary axis. We are looking for values of $\lambda$ where $\kappa_{4}^{-}$coincides with $\kappa_{3}^{-}$, when $\kappa_{3}^{-}$crosses into the essential spectrum, and where $\kappa_{4}^{+}$coincides with $\kappa_{1}^{+}$as $\kappa_{1}^{+}$enters the essential spectrum ahead of $\kappa_{3}^{+}$. In our case this occurs when $\lambda^{*}=-c^{2} / 4$. That $\lambda^{*}$ corresponds to a double root of the linear dispersion relations $d_{ \pm}(\lambda, v)=0$ at the asymptotic rest states at $\pm \infty$.

According to [22, Sect. 3.3], the sign of $\lambda^{*}<0$ indicates that the linear system convects perturbations in a preferred direction. The group velocity, or the direction in which the linear system convects, is given by $-(\mathrm{d} \operatorname{Im} \lambda) /(\mathrm{d} v)=-c<0$, thus the convection is toward $-\infty$. To stabilize the situation at $-\infty$, it is convenient to introduce exponential weights with positive rates. An exponential weight with a positive rate pushes the essential spectrum to the left of the imaginary axis [23, Sect. 4.7]. This is reflected in the dynamics of spatial eigenvalues in the following way. For $\lambda$ outside the essential spectrum the set $\sigma^{u}(\lambda)$ of unstable spatial eigenvalues is separated from the stable set $\sigma^{s}(\lambda)$ by the imaginary axis. When $\lambda$ crosses the imaginary axis $\sigma^{s}(\lambda)$ are $\sigma^{u}(\lambda)$ still separated, in this case by the vertical line $\operatorname{Re} \lambda=-d$ for some $d>0$ (see Fig. 2.1). The double root of the dispersion relation at $\lambda^{*}$ discussed above is the point where this separation ceases to exist.

2.2. Essential spectrum in the exponentially weighted spaces. The eigenvalue problem for the weighted variable $\tilde{P}=e^{\alpha \xi} P$ reads

$$
\begin{aligned}
& \lambda \tilde{p}_{1}=\partial_{\xi \xi} \tilde{p}_{1}+(c-2 \alpha) \partial_{\xi} \tilde{p}_{1}+\Omega\left(h_{1}\right) \tilde{p}_{2}+\left(h_{2} \Omega_{h_{1}}\left(h_{1}\right)-c \alpha+\alpha^{2}\right) \tilde{p}_{1}, \\
& \lambda \tilde{p}_{2}=\varepsilon \partial_{\xi \xi} \tilde{p}_{2}+(c-2 \alpha) \partial_{\xi} \tilde{p}_{2}+\left(\varepsilon \alpha^{2}-c \alpha-\beta \Omega\left(h_{1}\right)\right) \tilde{p}_{2}-\beta h_{2} \Omega_{h_{1}}\left(h_{1}\right) \tilde{p}_{1} .
\end{aligned}
$$



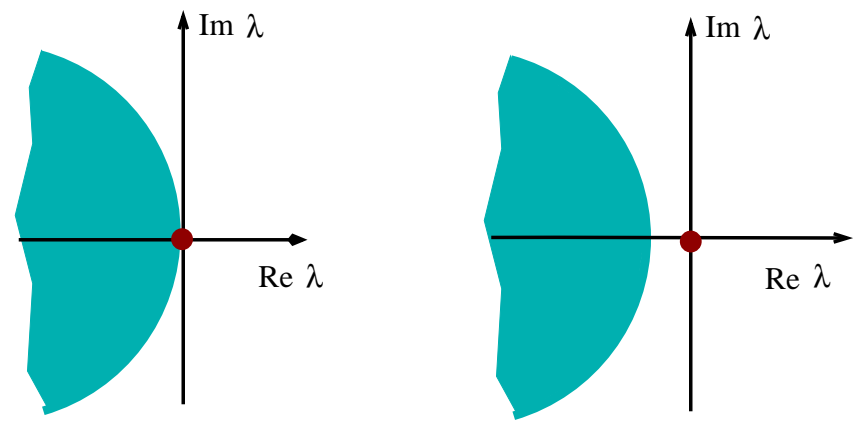

FIGURE 2.2. The rightmost boundary of the essential spectrum before (left panel) and after (right panel) applying exponential weights with positive rate.

In the notations of the previous section, if we write system (2.5) as a first order ODE, its right hand side is determined by the matrix $M(\lambda, \xi)+\alpha I$. The asymptotic matrices $M_{ \pm}(\lambda)+\alpha I$ have imaginary eigenvalues for values of $\lambda$ strictly to the left of the essential spectrum of $M_{ \pm}(\lambda)$. The boundaries of the essential spectrum of $(2.5)$ due to the behavior at $+\infty$ are given by the curves

$$
\begin{aligned}
& \left\{\lambda=-\varepsilon \nu^{2}+(c-2 \alpha) i v+\varepsilon \alpha^{2}-c \alpha ; \nu \in \mathbb{R}\right\} \\
& \cup\left\{\lambda=-v^{2}+(c-2 \alpha) i v-c \alpha+\alpha^{2} ; \nu \in \mathbb{R}\right\},
\end{aligned}
$$

and due to the behavior at $-\infty$ by the curves

$$
\begin{aligned}
& \left\{\lambda=-\varepsilon \nu^{2}+(c-2 \alpha) i v+\varepsilon \alpha^{2}-c \alpha-\beta e^{-\beta} ; \nu \in \mathbb{R}\right\} \\
& \cup\left\{\lambda=-v^{2}+(c-2 \alpha) i v-c \alpha+\alpha^{2} ; \nu \in \mathbb{R}\right\}
\end{aligned}
$$

The essential spectrum is bounded by the curves (2.6) and (2.7) and includes those. Choosing any $0<\alpha<\frac{c}{2}$ will make $-c \alpha+\alpha^{2}<0$, and therefore will push the essential spectrum to the left of the imaginary axis.

For small $\alpha>0$, the essential spectrum of the right hand side of (2.5) is strictly to the left of the imaginary axis. As to the discrete spectrum, it is known [12, Sect. 1.4] that exponential weights do not create new eigenvalues, but can remove them if the rate of convergence of the eigenfunction is slower than the one required by the exponentially weighted spaces. This can cause potentially dangerous loss of information in situations when, in the space without the weight, unstable $(\operatorname{Re} \lambda>0)$ or marginally unstable $(\operatorname{Re} \lambda=0)$ eigenvalues are present. In the present case, we only consider parameter regimes when there are no such eigenvalues except for a simple eigenvalue $\lambda=0$. The eigenvalue at the origin 
survives if the weight is such that the derivative of the front (the eigenfunction of $\lambda=0$ ) belongs to the weighted space. The weight is chosen based on the information about the exponential decay rates of the derivative of the front to zero at $\pm \infty$ that are the same as the decay rates of the front to its asymptotic rest states.

\subsection{Linear stability.}

Proposition 2.4. Assume that $\beta$ is such that the linear operator $\mathcal{M}_{0}$ defined by (2.1) does not have nonzero isolated eigenvalues with non-negative real parts except for the simple eigenvalue at the origin. The spectrum $\sigma\left(\mathcal{M}_{0}\right)$ then satisfies

$$
\sigma\left(\mathcal{M}_{0}\right) \subset\{\lambda \in \mathbb{C} ; \operatorname{Re} \lambda<0\} \cup\{0\} .
$$

Moreover, for any $0<\alpha<c / 2$ there exists $v_{0}>0$ such that

$$
\sigma\left(\mathcal{M}_{\alpha}\right) \subset\left\{\lambda \in \mathbb{C} ; \operatorname{Re} \lambda<-v_{0}\right\} \cup\{0\} .
$$

By the properties of sectorial operators from [14] the front in the co-moving frame is then linearly stable in the exponentially weighted space $H_{\alpha}^{1}$.

Proof. Under this assumption on $\beta$, the bound (2.8) is defined by the location of the rightmost boundary of the essential spectrum of $\mathcal{M}_{0}$ given by (2.4).

The exponential weight with any sufficiently small positive rate $\alpha>0$ pushes the essential spectrum to the left of the imaginary axis. In addition to the isolated eigenvalues of $\mathcal{M}_{0}$ in the open left-half plane that persist as eigenvalues of $\mathcal{M}_{\alpha}$, it is possible that isolated eigenvalues of the operator $\mathcal{M}_{\alpha}$ appear in the exposed area. The constant $-v_{0}$ is defined as the maximum of the real parts of the eigenvalues of $\mathcal{M}_{\alpha}$ and the rightmost point of the shifted essential spectrum.

For the proof of the nonlinear stability result, depending on the relation between $c$ and $\beta$, a more precise assumption on the upper bound for $\alpha$ is necessary, as described in the following lemma.

Lemma 2.5. There exists $\tilde{\alpha}>0$ such that for any $0<\alpha \leq \tilde{\alpha}$ the following is true about the front $H=\left(h_{1}, h_{2}\right)$ : For any fixed $b$

- $h_{2}(\xi+b) \mathrm{e}^{-\alpha \xi} \in C^{1}$,

- $H_{\xi}(\xi+b) \mathrm{e}^{-\alpha \xi} \in H^{1}$,

- $H_{\xi}(\xi+b) \mathrm{e}^{\alpha \xi} \in H^{1}$.

Proof. The geometric construction [9] shows that the front is formed as a transversal intersection of the unstable manifold of the burned state and the stable manifold of the unburned state. The component $h_{2}$ of the front, and the first and second order derivatives of $h_{1}$ and $h_{2}$ are not only uniformly bounded, but also approach 0 at $-\infty$ exponentially fast, let us say at rate $\gamma_{-}$that depends on $\beta, c$ and $\varepsilon$. At $+\infty$ the front exponentially converges to its rest state at the rate $-\gamma_{+}$, $\gamma_{+}=\gamma_{+}(\beta, c, \varepsilon)$. At the same rates the derivative of the front converges to 0 at $\pm \infty$. If $\alpha \leq \gamma_{ \pm}$than then the statements of the lemma hold. 
In what follows we assume that

$$
0<\alpha<\hat{\alpha}=\min \left\{\frac{c}{2}, \tilde{\alpha}\right\} .
$$

\section{NOnlinear STAbility in THE WeIghted SPaCES}

This section contains the proof of Theorem 1.3.1. The goal is to show that the front, when considered in the co-moving frame, is asymptotically stable in the weighted norm for the class of initial perturbations that are small in $C^{1}$ and, in addition, exponentially localized at $x=\infty$. The proof of Theorem 1.3.1 is divided in two parts. First we will show that suitable a priori estimates imply the nonlinear stability of the front in the co-moving frame in appropriate exponentially weighted norms. The a priori estimates are established afterward.

3.1. A priori estimates imply nonlinear stability. Solutions initially close to the front are expected to converge to an appropriate translate of the front but not necessarily to the front $H$ itself. To capture this behavior, we introduce a timedependent spatial shift function $q(t)$ in the argument of the front $H$ and write solutions to (1.3) as

$$
U(\xi, t)=H(\xi-q(t))+V(\xi, t) .
$$

Because of the translational invariance, one may assume, without loss of generality, that $q(0)=0$. The decomposition (3.1) can be made unique by requiring that the perturbation $V=\left(v_{1}, v_{2}\right)$ is orthogonal, in an appropriate way discussed below, to the one-dimensional subspace spanned by the derivative of the front.

The perturbation $V=\left(v_{1}, v_{2}\right)$ of the front satisfies the system

$$
\begin{aligned}
\partial_{t} v_{1}=\partial_{\xi \xi} v_{1}+c \partial_{\xi} v_{1}+\dot{q}(t) h_{1}^{\prime}(\xi-q(t)) & \\
+\left(h_{2}(\xi-q(t))+v_{2}\right) \Omega( & \left.h_{1}(\xi-q(t))+v_{1}\right) \\
& -h_{2}(\xi-q(t)) \Omega\left(h_{1}(\xi-q(t))\right), \\
\partial_{t} v_{2}=\varepsilon \partial_{\xi \xi} v_{2}+c \partial_{\xi} v_{2}+\dot{q}(t) h_{2}^{\prime}(\xi-q(t)) & -\beta\left[\left(h_{2}(\xi-q(t))+v_{2}\right) \Omega\left(h_{1}(\xi-q(t))+v_{1}\right)\right. \\
- & \left.-h_{2}(\xi-q(t)) \Omega\left(h_{1}(\xi-q(t))\right)\right]
\end{aligned}
$$

with initial data $v_{1}(\xi, 0)=v_{1}^{0}(\xi), v_{2}(\xi, 0)=v_{2}^{0}(\xi)$ and $q(0)=0$. For the nonlinear terms we will use the representation obtained in [10],

$$
\begin{aligned}
& \left(h_{2}(\xi-q(t))+v_{2}\right) \Omega\left(h_{1}(\xi-q(t))+v_{1}\right) \\
& \quad-h_{2}(\xi-q(t)) \Omega\left(h_{1}(\xi-q(t))\right)-h_{2}(\xi) \Omega_{h_{1}}\left(h_{1}(\xi)\right) v_{1}-\Omega\left(h_{1}(\xi)\right) v_{2} \\
& =v_{1}\left(v_{2} f_{1}+h_{2}(\xi-q(t)) f_{2}\right),
\end{aligned}
$$


where

$$
\begin{aligned}
f_{1} & =f_{1}(\xi, q(t), t) \\
& =\int_{0}^{1} \Omega_{h_{1}}\left(h_{1}(\xi-q(t))+s v_{1}(\xi, t)\right) \mathrm{d} s, \\
f_{2} & =f_{2}(\xi, q(t), t) \\
& =\int_{0}^{1}\left[\Omega_{h_{1}}\left(h_{1}(\xi-q(t))+s v_{1}(\xi, t)\right)-\Omega_{h_{1}}\left(h_{1}(\xi-q(t))\right)\right] \mathrm{d} s .
\end{aligned}
$$

Using the notation

$$
\begin{aligned}
\mathcal{L}\left(\partial_{\xi}\right) & =\left(\begin{array}{cc}
\partial \xi \xi+c \partial_{\xi} & 0 \\
0 & \varepsilon \partial \xi \xi+c \partial_{\xi}
\end{array}\right), \\
\mathcal{R}(\xi) & =\left(\begin{array}{cc}
h_{2}(\xi) \Omega_{h_{1}}\left(h_{1}(\xi)\right) & \Omega\left(h_{1}(\xi)\right) \\
-\beta h_{2}(\xi) \Omega_{h_{1}}\left(h_{1}(\xi)\right) & -\beta \Omega\left(h_{1}(\xi)\right)
\end{array}\right), \\
\Delta \mathcal{R}(\xi, \cdot) & =\mathcal{R}(\xi-\cdot)-\mathcal{R}(\xi), \\
\mathcal{N}(V) & =v_{2} f_{1}+h_{2}(\xi-q(t)) f_{2},
\end{aligned}
$$

we rewrite (3.2) as

$\partial_{t} V=\mathcal{L}\left(\partial_{\xi}\right) V+\mathcal{R}(\xi) V+\Delta \mathcal{R}(\xi, q(t)) V+\left(\begin{array}{c}1 \\ -\beta\end{array}\right) \mathcal{N}(V) v_{1}+\dot{q}(t) H^{\prime}(\xi-q(t))$.

The weighted solution

$$
W(\xi, t)=\left(w_{1}(\xi, t), w_{2}(\xi, t)\right)=e^{\alpha \xi} V(\xi, t),
$$

satisfies the system

(3.3) $\partial_{t} W=\Lambda_{\alpha} W+\Delta \mathcal{R}(\xi, q(t)) W+\left(\begin{array}{c}1 \\ -\beta\end{array}\right) \mathcal{N}(V) w_{1}+\dot{q}(t) e^{\alpha \xi} H^{\prime}(\xi-q(t))$, where

$$
\Lambda_{\alpha}=\mathcal{L}\left(\partial_{\xi}-\alpha\right)+\mathcal{R}(\xi) .
$$

Throughout the remainder of the proof, $\alpha$ is as in (2.9) and $v_{0}$ is as in Proposition 2.4: it is known then that $\lambda=0$ is a simple isolated eigenvalue of $\Lambda_{\alpha}$ with eigenfunction $e^{\alpha \xi} H^{\prime}(\xi)$ and the rest of the spectrum has real parts less than $\nu_{0}$. 
Due to the assumption on $\beta$, the eigenspace of $\Lambda_{\alpha}$ corresponding to the zero eigenvalue is one-dimensional. Define $\mathcal{P}_{\alpha}^{\mathrm{c}}: H^{1} \times H^{1} \rightarrow H^{1} \times H^{1}$ to be the central spectral projection, that is the projection on that one-dimensional eigenspace, and denote by $\mathcal{P}_{\alpha}^{s}=1-\mathcal{P}_{\alpha}^{c}$ the complementary projection onto the stable eigenspace.

The following lemma is a direct consequence of Proposition 2.4.

Lemma 3.2. Assume that $v_{0}$ as in Proposition 2.4 and $\hat{\alpha}$ is defined in (2.9). For $0<\alpha<\hat{\alpha}$, there is a constant $K_{0}>0$ such that the following is true about the analytic semigroup $\mathrm{e}^{\mathcal{P}_{\alpha}^{s} \Lambda_{\alpha} t}$ generated by $\mathcal{P}_{\alpha}^{\mathrm{s}} \Lambda_{\alpha}$,

$$
\left\|\mathrm{e}^{\mathcal{P}_{\alpha}^{\varsigma} \Lambda_{\alpha} t}\right\|_{H^{1} \rightarrow H^{1}} \leq K_{0} \mathrm{e}^{-v_{0} t}, \quad t \geq 0 .
$$

The uniqueness of $q(t)$ is provided by requiring that $\mathcal{P}_{\alpha}^{\mathrm{c}} W(t)=0$ for all $t$ for which the decomposition (3.1) exists, in other words, $W(t) \in \operatorname{Range}\left(\mathcal{P}_{\alpha}^{s}\right)$ for all $t$. Let $\psi^{c}$ span the kernel of the operator conjugate to $\Lambda_{\alpha}$ and assume that $\psi^{c}$ is appropriately normalized. Applying $\mathcal{P}_{\alpha}^{c}$ and $\mathcal{P}_{\alpha}^{\mathrm{s}}$ to (3.3), one obtains the evolution system for $V=\left(v_{1}, v_{2}\right), W=\left(w_{1}, w_{2}\right)$ and $q$

$$
\begin{aligned}
(3.5) \partial_{t} V= & \mathcal{L}\left(\partial_{\xi}\right) V+\mathcal{R}(\xi) V+\Delta \mathcal{R}(\xi, q(t)) V \\
& +\left(\begin{array}{c}
1 \\
-\beta
\end{array}\right) v_{1}\left(v_{2} f_{1}+h_{2}(\xi-q(t)) f_{2}\right)+\dot{q}(t) H^{\prime}(\xi-q(t)), \\
\text { (3.6) } \partial_{t} W= & \mathcal{P}_{\alpha}^{\mathrm{s}} \Lambda_{\alpha} W+\mathcal{P}_{\alpha}^{\mathrm{s}} \Delta \mathcal{R}(\xi, q(t)) W \\
& +\mathcal{P}_{\alpha}^{\mathrm{s}}\left(\begin{array}{c}
1 \\
-\beta
\end{array}\right) w_{1}\left(v_{2} f_{1}+h_{2}(\xi-q(t)) f_{2}\right)+\mathcal{P}_{\alpha}^{\mathrm{s}} \dot{q}(t) e^{\alpha \xi} H^{\prime}(\xi-q(t)), \\
(3.7) \dot{q}(t)= & -\left\langle\psi^{c}, e^{\alpha \xi} H^{\prime}(\xi-q(t))\right\rangle_{L^{2}}^{-1}\left\langle\psi^{c}, \Delta \mathcal{R}(\xi, q(t)) W\right. \\
& \left.+\left(\begin{array}{c}
1 \\
-\beta
\end{array}\right) w_{1}\left(v_{2} f_{1}+h_{2}(\xi-q(t)) f_{2}\right)\right\rangle_{L^{2}} .
\end{aligned}
$$

It is easy to see that the linear part of the right-hand sides in (3.5)-(3.6) is a sectorial operator on $H^{1} \times H^{1} \times H^{1} \times H^{1}$ with the dense domain $H^{3} \times H^{3} \times H^{3} \times H^{3}$. The nonlinearity is a smooth mapping from $\mathcal{Y}:=H^{1}\left(\mathbb{R}, \mathbb{R}^{4}\right) \times \mathbb{R}$ into itself, and there is a constant $K_{1}$ such that

$$
\|\Delta \mathcal{R}(\cdot,-q)\|_{H^{1}}+\|\mathcal{N}(V)\|_{H^{1}} \leq K_{1}\left(|q|+\|V\|_{H^{1}}\right) .
$$

Since $\left\langle\psi^{c}, e^{\alpha \xi} H^{\prime}(\xi-q(t))\right\rangle_{L^{2}}=1+O(q)$ we also have that

$$
|\dot{q}| \leq K_{1}\left(|q|+\|V\|_{H^{1}}\right)\|W\|_{H^{1}}
$$

for all $(V, W, q) \in \mathcal{Y}$ with norm less than one, for example. We therefore have the methods introduced in [14] at our disposal that give local existence and uniqueness of solutions for initial data in $\mathcal{Y}$ as well as continuous dependence on initial conditions, thus proving local existence and uniqueness of decomposition (3.1). 
Based on these arguments it is reasonable to claim that, for each given $0<$ $\delta^{*} \leq 1$, there exists a $\rho>0$ and a time $T>0$ such that decomposition (3.1) exists for $0 \leq t<T$ with

$$
|q(t)|+\|V(t)\|_{H^{1}} \leq \delta^{*}
$$

provided $\left\|V^{0}\right\|_{H^{1}} \leq \rho$. Let $T_{\max }=T_{\max }\left(\delta^{*}\right)$ be the maximal time for which (3.10) holds. The following lemma is proved essentially in the same way as in [11], where a front exhibited by a system that consists of a Swift-Hohenberg equation coupled nonlinearly to a Chaffee-Infante equation is studied and the nature of its nonlinear stability is analyzed.

Lemma 3.3. Pick $v$ with $0<v<v_{0}$ and $\hat{\delta}>0$ so that

$$
\frac{K_{0} K_{1}\left(1+K_{0}\right)}{v_{0}-v} \hat{\delta}<\frac{1}{2}
$$

then there are positive constants $K_{2}$ and $K_{3}$ such that for any $0<\delta^{*} \leq \hat{\delta}$

$$
\|W(t)\|_{H^{1}} \leq K_{2} \mathrm{e}^{-v t}\|W(0)\|_{H^{1}}, \quad|q(t)| \leq K_{3}\|W(0)\|_{H^{1}}
$$

for all $0 \leq t<T_{\max }\left(\delta^{*}\right)$ and any solution that satisfies (3.10). If $T_{\max }\left(\delta^{*}\right)=\infty$, then there is a constant $q_{*} \in \mathbb{R}$ such that

$$
\left|q(t)-q_{*}\right| \leq \frac{K_{1} K_{2}}{v_{0}} \mathrm{e}^{-v t}\|W(0)\|_{H^{1}}
$$

for $t \geq 0$.

To complete the proof of Theorem 1.3.1 once Lemma 3.3 has been proved, it suffices to establish a priori estimates and show that $V(t)$ always stays small enough and therefore that $T_{\max }=\infty$, if $\hat{\delta}$ satisfies (3.11).

Proof. The variation-of-constants formula applied to (3.6) gives

$$
\begin{aligned}
W(t)=\mathrm{e}^{\mathcal{P}_{\alpha}^{s} \Lambda_{\alpha} t} W(0)+\int_{0}^{t} \mathrm{e}^{\mathcal{P}_{\alpha}^{s} \Lambda_{\alpha}(t-s)} \mathcal{P}_{\alpha}^{s} & \\
\times & {\left[\Delta \mathcal{R}(\xi, q(s)) W+\left(\begin{array}{c}
1 \\
-\beta
\end{array}\right) w_{1}\left(v_{2} f_{1}+h_{2}(\xi-q(t)) f_{2}\right)\right.} \\
& \left.+\dot{q}(s) H_{\xi}(\xi-q(s)) \mathrm{e}^{\alpha \xi}\right] \mathrm{d} s .
\end{aligned}
$$

Using estimates (3.4), (3.8) and (3.10) we obtain 


$$
\begin{aligned}
\|W(t)\|_{H^{1}} \leq K_{0} \mathrm{e}^{-v_{0} t}\|W(0)\|_{H^{1}}+K_{0} \int_{0}^{t} \mathrm{e}^{-v_{0}(t-s)} & \\
& \times\left[K_{1} \delta^{*}\|W(s)\|_{H^{1}}+|\dot{q}(s)|\left\|H_{\xi}(\xi-q(s)) \mathrm{e}^{\alpha \xi}\right\|_{H^{1}}\right] \mathrm{d} s .
\end{aligned}
$$

It follows from Lemma 2.5 that there exists a $\tilde{K}>0$ such that $\left\|H_{\xi}(\xi-q(s)) \mathrm{e}^{\alpha \xi}\right\|_{H^{1}} \leq \tilde{K}$. Therefore due to (3.9) one has

$$
\begin{aligned}
\|W(t)\|_{H^{1}} \leq & K_{0} \mathrm{e}^{-v_{0} t}\|W(0)\|_{H^{1}} \\
& +K_{0} K_{1}(1+\tilde{K}) \delta^{*} \int_{0}^{t} \mathrm{e}^{-v_{0}(t-s)}\|W(s)\|_{H^{1}} \mathrm{~d} s
\end{aligned}
$$

for $0<t<T_{\max }$. For any $v$ such that $0<v<v_{0}$, we define

$$
M(T):=\sup _{0 \leq t \leq T} \mathrm{e}^{v t}\|W(t)\|_{H^{1}},
$$

where $0 \leq T \leq T_{\max }$ and $T$ is finite. Equation (3.13) gives

$$
\begin{aligned}
& \mathrm{e}^{v t}\|W(t)\|_{H^{1}} \\
& \quad \leq K_{0} \mathrm{e}^{-\left(v_{0}-v\right) t}\|W(0)\|_{H^{1}}+K_{0} K_{1}(1+\tilde{K}) \delta^{*} \int_{0}^{t} \mathrm{e}^{-\left(v_{0}-v\right)(t-s)} \mathrm{e}^{v s}\|W(s)\|_{H^{1}} \mathrm{~d} s \\
& \quad \leq K_{0}\|W(0)\|_{H^{1}}+2 K_{0} K_{1}(1+\tilde{K}) \delta^{*} M(T) \int_{0}^{t} \mathrm{e}^{-\left(v_{0}-v\right)(t-s)} \mathrm{d} s,
\end{aligned}
$$

which yields

$$
\begin{aligned}
M(T) & \leq K_{0}\|W(0)\|_{H^{1}}+\frac{K_{0} K_{1}(1+\tilde{K}) \delta^{*}}{v_{0}-v} M(T) \\
& \leq K_{0}\|W(0)\|_{H^{1}}+\frac{K_{0} K_{1}(1+\tilde{K}) \delta^{*}}{v_{0}-v} M(T) .
\end{aligned}
$$

Assume $\hat{\delta}>0$ to be such that (3.11) holds; then there is a constant $K_{2}>0$ such that for any $0<\delta^{*} \leq \hat{\delta}$

$$
\sup _{0 \leq t \leq T} \mathrm{e}^{v t}\|W(t)\|_{H^{1}} \leq K_{2}\|W(0)\|_{H^{1}}
$$

and therefore

$$
\|W(t)\|_{H^{1}} \leq K_{2} \mathrm{e}^{-v t}\|W(0)\|_{H^{1}}
$$


for $0 \leq t \leq T$ as desired. Estimate (3.14) combined with (3.9) yields

$$
|\dot{q}(t)| \leq K_{1} K_{2} \delta^{*} \mathrm{e}^{-v t}\|W(0)\|_{H^{1}}
$$

for $0 \leq t \leq T$. An estimate for $q(t)$ is obtained from the equation

$$
q(t)=q(s)+\int_{S}^{t} \dot{q}(\tau) \tau,
$$

from where due to (3.15) it follows that at $s=0$

$$
|q(t)| \leq \int_{0}^{t}|\dot{q}(\tau)| \mathrm{d} \tau \leq K_{1} K_{2} \delta^{*}\|W(0)\|_{H^{1}} \int_{0}^{t} \mathrm{e}^{-v \tau} \mathrm{d} \tau \leq \frac{K_{1} K_{2} \delta^{*}}{v}\|W(0)\|_{H^{1}} .
$$

We recall that $0<\delta^{*}<1$. The desired estimate is then obtained by setting $K_{3}=K_{1} K_{2} / \nu$ (notice that $K_{3}$ then is independent of $\delta^{*}$ )

$$
|q(t)| \leq K_{3}\|W(0)\|_{H^{1}}
$$

for $0 \leq t \leq T$.

Lastly, if $T_{\max }=\infty$, then (3.14), (3.9) and (3.17) are valid for all times since the constants $K_{2}$ and $K_{3}$ do not depend upon $T$ or on $\delta^{*}$. Thus, equation (3.15) implies that the limit $\mathcal{q}_{*}=\lim _{t \rightarrow \infty} q(t)$ exists, and (3.17) shows that $\left|\mathcal{q}_{*}\right| \leq$ $K_{3}\|W(0)\|_{H^{1}}$. Therefore, in the limit $s \rightarrow \infty$, (3.16) yields

$$
q(t)=q_{*}+\int_{\infty}^{t} q^{\prime}(\tau) \mathrm{d} \tau,
$$

which together with (3.15) give the estimate (3.12).

3.4. Establishing the necessary a priori estimates. To complete the proof of Theorem 1.3.1, it suffices to show that, for $0<\delta^{*} \leq \hat{\delta}$ with $\hat{\delta}$ as in Lemma 3.3, there exists a $\rho>0$ such that

$$
|q(t)|+\|V(t)\|_{H^{1}} \leq \delta^{*}
$$

for all $t \geq 0$ provided

$$
\left\|V^{0}\right\|_{H^{1}}+\|W(0)\|_{H^{1}} \leq \rho .
$$

Throughout this section, we assume that $q(0)=0, V^{0} \in H^{1}$, and $W(0)=\mathrm{e}^{\alpha \xi} V^{0}$ is also in $H^{1}$.

Assume $\left(v_{1}, v_{2}, w_{1}, w_{2}, q\right)$ satisfy the system (3.5)-(3.7). We first analyze the equation for the perturbation $v_{2}$ to the concentration component of the front. 
Function $v_{2}$ is a solution of the equation

$$
\begin{aligned}
\partial_{t} v_{2}=\varepsilon \partial_{\xi \xi} v_{2}+c \partial \xi v_{2}-\beta \mathrm{e}^{-\beta} v_{2} & \\
+\beta\left[\mathrm{e}^{-\beta}-\Omega\left(h_{1}(\xi-q(t))\right)\right] & v_{2}-\beta h_{2} \Omega_{h_{1}}\left(h_{1}(\xi-q(t))\right) v_{1} \\
& -\beta v_{1}\left(v_{2} f_{1}+h_{2} f_{2}\right)+\dot{q}(t) h_{2}^{\prime}(\xi-q(t)) .
\end{aligned}
$$

Recall that the rate $\alpha$ in the exponential weight is a number from the interval $(0, \tilde{\alpha})$ chosen according to Lemma 2.5 . The following simple lemma follows from the properties of the front $H$.

Lemma 3.5. Assume that $\alpha$ is as in Lemma 2.5. There exist then constants $M_{1}, M_{2}, M_{3}, M_{4}, M_{5}, M_{6}, M_{7}>0$ that are independent of $\alpha$ and are such that the following is true for any $\xi \in \mathbb{R}$ and $t \geq 0$ :

- $\left\|\left[\mathrm{e}^{-\beta}-\Omega\left(h_{1}(\xi-q(t))\right)\right] \mathrm{e}^{-\alpha \xi}\right\|_{C^{1}} \leq M_{1}$,

- $\| h_{2}(\xi-q(t)) \Omega_{h_{1}}\left(h_{1}(\xi-q(t)) \mathrm{e}^{-\alpha \xi} \|_{C^{1}} \leq M_{2}\right.$,

- $\left\|h_{2}(\xi-q(t)) f_{2} \mathrm{e}^{-\alpha \xi}\right\|_{H^{1}} \leq M_{3}\left(1+\left\|v_{1}\right\|_{H^{1}}\right) / 2 \leq M_{3}$, for $t<T_{\max }$,

- $\left\|f_{1}(\xi, t)\right\|_{H^{1}} \leq M_{4}\left(1+\left\|v_{1}\right\|_{H^{1}}\right) / 2 \leq M_{4}$, for $t<T_{\max }$,

- $\left\|\Omega\left(h_{1}(\xi)\right)\right\|_{C^{1}} \leq M_{5}$,

- $\left\|h_{1}^{\prime}(\xi)\right\|_{C^{1}} \leq M_{6}$,

- $\left\|h_{2}^{\prime}(\xi)\right\|_{C^{1}} \leq M_{7}$,

where $C^{1}$ norm is taken with respect to $\xi$.

Proof. The first bound follows from the fact that the $h_{1}$-component of the front approaches $1 / \beta$ at $\xi \rightarrow-\infty$ exponentially fast. Therefore $\Omega\left(h_{1}(\xi-q(t))\right)=$ $\mathrm{e}^{1 / h_{1}(\xi-q(t))}$ approaches $\mathrm{e}^{-\beta}$ faster than any fixed exponential rate. The derivative

$$
\begin{aligned}
\partial \xi\left[\mathrm{e}^{-\beta}-\Omega\right. & \left.\left(h_{1}(\xi-q(t))\right)\right] \mathrm{e}^{-\alpha \xi} \\
& =-\Omega_{h_{1}}\left(h_{1}(\xi-q(t))\right) h_{1}^{\prime} \mathrm{e}^{-\alpha \xi}-\alpha\left[\mathrm{e}^{-\beta}-\Omega\left(h_{1}(\xi-q(t))\right)\right] \mathrm{e}^{-\alpha \xi}
\end{aligned}
$$

is bounded by Lemma 2.5 .

The second and the third bounds are also consequences of Lemma 2.5.

The bounds for $f_{1}$ and $\Omega\left(h_{1}\right)$ follow from their definitions.

The inequalities $h_{2}^{\prime}>0$ and $h_{1}^{\prime}<0$ have been obtained in [9]. In the same style upper bounds for $h_{2}^{\prime}$ and $h_{1}^{\prime}$ can be obtained. The front is the unique solution of the ODE

$$
\begin{aligned}
h_{1}^{\prime \prime}+c h_{1}^{\prime} & =-h_{2} \Omega\left(h_{1}\right), \\
\varepsilon h_{2}^{\prime \prime}+c h_{2}^{\prime} & =\beta h_{2} \Omega\left(h_{1}\right)
\end{aligned}
$$

that satisfies the boundary conditions: $\left(h_{1}, h_{2}\right) \rightarrow(1 / \beta, 0)$ as $\xi \rightarrow-\infty$, and $\left(h_{1}, h_{2}\right) \rightarrow(0,1)$ as $\xi \rightarrow \infty$. The system (3.19) can be effectively integrated 
from $-\infty$ to $\xi$ :

$$
\begin{aligned}
h_{1}^{\prime}+c\left(h_{1}-\frac{1}{\beta}\right) & =-\int_{-\infty}^{\xi} h_{2}(z) \Omega\left(h_{1}(z)\right) \mathrm{d} z \\
\varepsilon h_{2}^{\prime}+c h_{2} & =\beta \int_{-\infty}^{\xi} h_{2}(z) \Omega\left(h_{1}(z)\right) \mathrm{d} z .
\end{aligned}
$$

For $\left(h_{1}, h_{2}\right)$ the boundary conditions at $+\infty$ are satisfied, therefore it is easy to see that

$$
\int_{-\infty}^{\infty} h_{2}(z) \Omega\left(h_{1}(z)\right) \mathrm{d} z=\frac{c}{\beta} .
$$

From (3.20) and (3.21), since both $h_{1}$ and $h_{2}$ are positive [9], the estimates follow:

$$
\begin{aligned}
& 0 \geq h_{1}^{\prime}=-c\left(h_{1}-\frac{1}{\beta}\right)-\int_{-\infty}^{\xi} h_{2}(z) \Omega\left(h_{1}(z)\right) \mathrm{d} z \geq-\frac{c}{\beta}, \\
& 0 \leq h_{2}^{\prime}=-\frac{c}{\varepsilon} h_{2}+\frac{\beta}{\varepsilon} \int_{-\infty}^{\xi} h_{2}(z) \Omega\left(h_{1}(z)\right) \mathrm{d} z \leq \frac{c}{\varepsilon} .
\end{aligned}
$$

For $h_{1}^{\prime \prime}$ and $h_{2}^{\prime \prime}$ uniform bounds are easy to obtain from (3.19).

Using Lemma 3.5 and the relation between $W$ and $V, W=\mathrm{e}^{\alpha \xi} V$, the equation (3.18) can be rewritten in an equivalent form

$$
\begin{aligned}
\partial_{t} v_{2}=\varepsilon \partial_{\xi \xi} v_{2}+c \partial_{\xi} v_{2}-\beta \mathrm{e}^{-\beta} v_{2}+\beta g_{1} w_{2} \\
-\beta g_{2} w_{1}-\beta v_{1} v_{2} f_{1}-\beta g_{3} w_{1}+\dot{q}(t) h_{2}^{\prime}(\xi-q(t))
\end{aligned}
$$

where

$$
\begin{aligned}
& g_{1}=\quad\left[\mathrm{e}^{-\beta}-\Omega\left(h_{1}(\xi-q(t))\right)\right] \mathrm{e}^{-\alpha \xi}, \quad\left\|g_{1}\right\|_{C^{1}} \leq M_{1}, \\
& g_{2}=h_{2}(\xi-q(t)) \Omega_{h_{1}}\left(h_{1}(\xi-q(t)) \mathrm{e}^{-\alpha \xi}, \quad\left\|g_{2}\right\|_{C^{1}} \leq M_{2}\right. \text {, } \\
& g_{3}=\quad h_{2}(\xi-q(t)) f_{2} \mathrm{e}^{-\alpha \xi}, \quad\left\|g_{3}\right\|_{C^{1}} \leq M_{3} \text { for } t<T_{\max } .
\end{aligned}
$$

The operator $L_{2}=\varepsilon \partial_{\xi \xi}+c \partial_{\xi}-\beta \mathrm{e}^{-\beta}$ is a sectorial operator on $H^{1}$. If the initial perturbation $v_{2}^{0}$ to the $v_{2}$-component of the front belongs to $H^{1}$ and is sufficiently small (as encoded in $|q(t)|+\left\|V^{0}\right\|_{H^{1}} \leq \delta^{*}<1$ ) then in finite time it will evolve to a function that is still in $H_{1}$. The operator $L_{2}$ generates an analytic semigroup $\mathrm{e}^{L_{2} t}$ for which standard semigroup estimates hold, i.e. there exist constants $0<$ $b<\beta \mathrm{e}^{-\beta}$ and $K_{4}>0$ such that

$$
\left\|\mathrm{e}^{L_{2} t}\right\|_{H^{1} \rightarrow H^{1}} \leq K_{4} \mathrm{e}^{-b t} .
$$


Bound (3.23) gives an additional restriction on $v$ from Lemma 3.3. From now on we assume that

$$
0<v<\min \left\{v_{0}, b\right\} .
$$

Applying the variation-of-constants formula to equation (3.22), one obtains

$$
\begin{aligned}
v_{2}=\mathrm{e}^{L_{2} t} & v_{2}^{0}+\int_{0}^{t} \mathrm{e}^{L_{2}(t-s)} \\
& \times\left[\beta g_{1} w_{2}-\beta\left(g_{2}+g_{3}\right) w_{1}-\beta v_{1} v_{2} f_{1}+\dot{q}(s) h_{2}^{\prime}(\xi-q(s))\right] \mathrm{d} s .
\end{aligned}
$$

The semigroup estimates (3.23), Lemma 3.5, and the bootstrap inequality (3.10) yield

(3.26) $\left\|v_{2}(t)\right\|_{H^{1}}$

$$
\begin{aligned}
\leq K_{4} \mathrm{e}^{-b t}\left\|v_{2}^{0}\right\|_{H^{1}} & +K_{4} \int_{0}^{t} \mathrm{e}^{-b(t-s)} \beta\left(M_{1}+M_{2}+M_{3}\right)\|W(s)\|_{H^{1}} \mathrm{~d} s \\
& +K_{4} \int_{0}^{t} \mathrm{e}^{-b(t-s)}\left[M_{7}|\dot{q}(s)|+\beta M_{4} \delta^{*}\left\|v_{2}(s)\right\|_{H^{1}}\right] \mathrm{d} s .
\end{aligned}
$$

Using in (3.26) the key inequalities, (3.14) for $\|W\|_{H^{1}}$ and (3.15) for $|\dot{q}|$, one then obtains

$$
\begin{aligned}
& \left.\left\|v_{2}(t)\right\|_{H^{1}} \leq K_{4} \mathrm{e}^{-b t}\left\|v_{2}^{0}\right\|_{H^{1}}+\beta M_{4} \delta^{*} K_{4} \int_{0}^{t} \mathrm{e}^{-b(t-s)}\left\|v_{2}(s)\right\|_{H^{1}}\right] \mathrm{d} s \\
& \quad+K_{4} \int_{0}^{t} \mathrm{e}^{-b(t-s)}\left(\beta\left(M_{1}+M_{2}+M_{3}\right) K_{2}+M_{7} K_{1}\right) \mathrm{e}^{-v s}\|W(0)\|_{H^{1}} \mathrm{~d} s .
\end{aligned}
$$

We multiply the inequality (3.27) by $\mathrm{e}^{(v / 2) t}$,

$$
\begin{aligned}
& \mathrm{e}^{(v / 2) t}\left\|v_{2}(t)\right\|_{H^{1}} \leq K_{4} \mathrm{e}^{-(b-v / 2) t}\left\|v_{2}^{0}\right\|_{H^{1}} \\
& \quad+K_{4}\left(\beta\left(M_{1}+M_{2}+M_{3}\right) K_{2}+M_{7} K_{1}\right)\|W(0)\|_{H^{1}} \mathrm{e}^{(v / 2) t} \int_{0}^{t} \mathrm{e}^{-b(t-s)} \mathrm{e}^{-v s} \mathrm{~d} s \\
& \quad+\delta^{*} \beta M_{4} K_{4} \int_{0}^{t} \mathrm{e}^{(v / 2) t} \mathrm{e}^{-b(t-s)}\left\|v_{2}(s)\right\|_{H^{1}} \mathrm{~d} s .
\end{aligned}
$$

Due to (3.24), $b-v / 2>0$. For the term in (3.28) that contains the first integral we find

$$
\begin{aligned}
\int_{0}^{t} \mathrm{e}^{(\nu / 2) t} \mathrm{e}^{-b(t-s)} \mathrm{e}^{-v s} \mathrm{~d} s & =\mathrm{e}^{-(b-v / 2) t} \int_{0}^{t} \mathrm{e}^{(b-v) s} \mathrm{~d} s \\
& =\mathrm{e}^{-(\nu / 2) t} \mathrm{e}^{-(b-v) t} \frac{\mathrm{e}^{(b-v) t}-1}{b-v}=\mathrm{e}^{-(\nu / 2) t} \frac{1-\mathrm{e}^{-(b-v) t}}{b-v},
\end{aligned}
$$


so the following bound is true:

$$
\int_{0}^{t} \mathrm{e}^{(v / 2) t} \mathrm{e}^{-b(t-s)} \mathrm{e}^{-v s} \mathrm{~d} s \leq \frac{1}{b-v} .
$$

For the second integral we have

$$
\begin{aligned}
\int_{0}^{t} \mathrm{e}^{(v / 2) t} \mathrm{e}^{-b(t-s)}\left\|v_{2}(s)\right\|_{H^{1}} \mathrm{~d} s & =\int_{0}^{t} \mathrm{e}^{-(b-v / 2)(t-s)} \mathrm{e}^{(v / 2) s}\left\|v_{2}(s)\right\|_{H^{1}} \mathrm{~d} s \\
& \leq \sup _{0<t<T_{\max }}\left\{\mathrm{e}^{(v / 2) t}\left\|v_{2}(t)\right\|_{H^{1}}\right\} \int_{0}^{t} \mathrm{e}^{-(b-v / 2)(t-s)} \mathrm{d} s \\
& =\sup _{0<t<T_{\max }}\left\{\mathrm{e}^{(v / 2) t}\left\|v_{2}(t)\right\|_{H^{1}}\right\} \frac{\mathrm{e}^{-(b-v / 2) t}-1}{-(b-v / 2)}
\end{aligned}
$$

therefore this integral can be controlled as

$$
\int_{0}^{t} \mathrm{e}^{(v / 2) t} \mathrm{e}^{-b(t-s)}\left\|v_{2}(s)\right\|_{H^{1}} \mathrm{~d} s \leq \sup _{0<t<T_{\max }}\left\{\mathrm{e}^{(\nu / 2) t}\left\|v_{2}(t)\right\|_{H^{1}}\right\} \frac{1}{b-v / 2}
$$

With bounds (3.29) and (3.30), inequality (3.28) becomes

$$
\begin{aligned}
& \mathrm{e}^{(v / 2) t}\left\|v_{2}(t)\right\|_{H^{1}} \\
& \leq K_{4}\left\|v_{2}^{0}\right\|_{H^{1}}+K_{4}\left(\beta\left(M_{1}+M_{2}+M_{3}\right) K_{2}+M_{7} K_{1}\right)\|W(0)\|_{H^{1}} \frac{1}{b-v} \\
& +\delta^{*} \beta M_{4} K_{4} \sup _{0<t<T_{\max }}\left\{\mathrm{e}^{(v / 2) t}\left\|v_{2}(t)\right\|_{H^{1}}\right\} \frac{1}{b-v / 2} .
\end{aligned}
$$

To continue we impose an additional condition on $\hat{\delta}$, and therefore on $\delta^{*}<\hat{\delta}$ :

$$
\hat{\delta} \leq \frac{1}{2}\left(\frac{\beta M_{4} K_{4}}{b-v / 2}\right)^{-1}=\frac{b-v / 2}{2 \beta M_{4} K_{4}} .
$$

Under this condition, one can take the supremum over all $0 \leq t \leq T_{\max }$ on both sides of (3.31), and obtain

$$
\begin{aligned}
\sup _{0<t<T_{\max }} & \left\{\mathrm{e}^{(v / 2) t}\left\|v_{2}(t)\right\|_{H^{1}}\right\} \\
& \leq 2 K_{4}\left\|v_{2}^{0}\right\|_{H^{1}}+2 K_{4}\left(\beta\left(M_{1}+M_{2}+M_{3}\right) K_{2}+M_{7} K_{1}\right)\|W(0)\|_{H^{1}} \frac{1}{\beta \mathrm{e}^{-\beta}-v} .
\end{aligned}
$$


Therefore for any $t<T_{\max }$,

$$
\begin{aligned}
& \left\|v_{2}(t)\right\|_{H^{1}} \\
& \leq \mathrm{e}^{-(v / 2) t}\left[2 K_{4}\left\|v_{2}^{0}\right\|_{H^{1}}+2 K_{4}\left(\beta\left(M_{1}+M_{2}+M_{3}\right) K_{2}+K_{1} M_{7}\right)\|W(0)\|_{H^{1}} \frac{1}{b-v}\right] .
\end{aligned}
$$

The following lemma is proved.

Lemma 3.6. Assume that $\alpha$ is such as in Lemma 2.5, $\hat{\delta}$ satisfies (3.11) and (3.32), and $v$ satisfies (3.24). There exist positive, independent of $\alpha$ constants $\rho *$ and $K_{5}$ such that for any $t \in\left[0, T_{\max }(\hat{\delta})\right)$

$$
\left\|v_{2}(t)\right\|_{H^{1}} \leq K_{5}\left[\left\|v_{2}^{0}\right\|_{H^{1}}+\|W(0)\|_{H^{1}}\right] \mathrm{e}^{-(v / 2) t},
$$

provided $\left\|V^{0}\right\|+\|W(0)\|_{H^{1}} \leq \rho^{*}$.

Lemma 3.6 says that the size of $\left\|v_{2}(t)\right\|_{H^{1}}$ within the time interval $\left[0, T_{\max }\right)$ can be controlled by choosing small enough initial perturbations $\left\|V^{0}\right\|_{H^{1}}$ and $\|W(0)\|_{H^{1}}$. Moreover, $\left\|v_{2}(t)\right\|_{H^{1}}$ decreases on $\left[0, T_{\max }\right)$ exponentially.

The next goal is to obtain a priori estimates for the temperature component $v_{1}$ of the perturbation to the front. The temperature $v_{1}$ satisfies the equation

$$
\begin{aligned}
\partial_{t} v_{1}=\partial_{\xi \xi} v_{1}+c \partial_{\xi} v_{1}+h_{2} \Omega_{h_{1}}\left(h_{1}(\xi-q(t))\right) v_{1} \\
\quad+\Omega\left(h_{1}(\xi-q(t))\right) v_{2}+v_{1}\left(v_{2} f_{1}+h_{2} f_{2}\right)+\dot{q}(t) h_{1}^{\prime}(\xi-q(t))
\end{aligned}
$$

Operator $L_{1}=\partial \xi \xi+c \partial_{\xi}$ is sectorial. It generates an analytic semigroup $\mathrm{e}^{L_{1} t}$ and, moreover, because of the estimates on the heat kernel, there exists a positive constant $K_{6}$

$$
\left\|\mathrm{e}^{L_{1} t}\right\|_{H^{1} \rightarrow H^{1}} \leq K_{6} .
$$

We rewrite Equation (3.34) in the following form

$$
\begin{aligned}
\partial_{t} v_{1}=L_{1} v_{1}+\left(h_{2} \Omega_{h_{1}}\left(h_{1}(\xi-q(t))\right)+h_{2} f_{2}\right) \mathrm{e}^{-\alpha \xi} w_{1} \\
+\left(\Omega\left(h_{1}(\xi-q(t))\right)+v_{1} f_{1}\right) v_{2}+\dot{q}(t) h_{1}^{\prime}(\xi-q(t)),
\end{aligned}
$$

and apply to it the variation-of-constants formula,

$$
\begin{aligned}
& v_{1}=\mathrm{e}^{L_{1} t} v_{1}^{0}+\int_{0}^{t} \mathrm{e}^{L_{1}(t-s)}\left[\left(h_{2} \Omega_{h_{1}}\left(h_{1}(\xi-q(t))\right)+h_{2} f_{2}\right) \mathrm{e}^{-\alpha \xi} w_{1}\right] \mathrm{d} s \\
& \left.+\int_{0}^{t} \mathrm{e}^{L_{1}(t-s)}\left[\left(\Omega\left(h_{1}(\xi-q(t))\right)\right)+v_{1} f_{1}\right) v_{2}+\dot{q}(t) h_{1}^{\prime}(\xi-q(t))\right] \mathrm{d} s .
\end{aligned}
$$


From Lemma 3.5 and semigroup estimates (3.35) it follows that

$$
\begin{aligned}
\left\|v_{1}(t)\right\|_{H^{1}} \leq & K_{6}\left\|v_{1}^{0}\right\|_{H^{1}}+K_{6}\left(M_{2}+M_{3}\right) \int_{0}^{t}\left\|w_{1}(s)\right\|_{H^{1}} \mathrm{~d} s \\
& +K_{6}\left(M_{5}+\delta^{*} M_{4}\right) \int_{0}^{t}\left\|v_{2}(s)\right\|_{H^{1}} \mathrm{~d} s+K_{6} M_{6} \int_{0}^{t}|\dot{q}(s)| \mathrm{d} s .
\end{aligned}
$$

In (3.37), we use inequality (3.14) to estimate the term containing $\left\|w_{1}\right\|_{H^{1}}$, and (3.15) to estimate the term containing $|\dot{q}(t)|$. The term with $\left\|v_{2}\right\|_{H^{1}}$ is controlled according to (3.33). The estimate (3.37) then implies

$$
\begin{aligned}
&\left\|v_{1}(t)\right\|_{H^{1}} \leq K_{6}\left\|v_{1}^{0}\right\|_{H^{1}}+K_{6}\left(M_{2}+M_{3}\right) K_{2}\|W(0)\|_{H^{1}} \int_{0}^{t} \mathrm{e}^{-v s} \mathrm{~d} s \\
&+K_{6}\left(M_{5}+\delta^{*} M_{4}\right) K_{5}\left[\left\|v_{2}^{0}\right\|_{H^{1}}+\|W(0)\|_{H^{1}}\right] \int_{0}^{t} \mathrm{e}^{-(v / 2) s} \mathrm{~d} s \\
&+K_{6} K_{1} K_{2} M_{6}\|W(0)\|_{H^{1}} \int_{0}^{t} \mathrm{e}^{-v s} \mathrm{~d} s .
\end{aligned}
$$

Evaluating the integrals on the right hand side of (3.38) one obtains

$$
\begin{aligned}
\left\|v_{1}(t)\right\|_{H^{1}} \leq K_{6}\left\|v_{1}^{0}\right\|_{H^{1}} & +K_{6}\left[\left(M_{2}+M_{3}\right) K_{2}+K_{6} K_{1} K_{2} M_{6}\right] \frac{1-\mathrm{e}^{v} t}{v}\|W(0)\|_{H^{1}} \\
& +K_{6}\left(M_{5}+\delta^{*} M_{4}\right) K_{5}\left[\left\|v_{2}^{0}\right\|_{H^{1}}+\|W(0)\|_{H^{1}}\right] \frac{2\left(1-\mathrm{e}^{v / 2} t\right)}{v},
\end{aligned}
$$

from where the next lemma follows.

Lemma 3.7. Under conditions of Lemma 3.6 there exists a positive constant $K_{7}$ such that for any $t \in\left[0, T_{\max }\right)$

$$
\left\|v_{1}(t)\right\|_{H^{1}} \leq K_{7}\left(\left\|V^{0}\right\|_{H^{1}}+\|W(0)\|_{H^{1}}\right) .
$$

The result on nonlinear stability in the weighted norm in Theorem 1.3.1 follows now from Lemmas 3.6 and 3.7. Indeed, the lemmas imply that if $\|W(0)\|_{H^{1}}$ and $\left\|V^{0}\right\|_{H^{1}}$ are sufficiently small, then (3.10) holds for all $t>0$ so that (3.14) and (3.17) are valid for all positive times.

Lemma 3.7 says that perturbations to the temperature component $v_{1}$ of the front, if initially sufficiently small in $H^{1}$-norm, stay bounded in $H^{1}$-norm. It is possible to show that if the initial perturbation additionally belongs to the space $L^{1}$ then $v_{1}$ also decays in time, but at an algebraic rate. This does not add to our nonlinear stability result, but shows that eventually the perturbed front converges to a translate of the same front, and not to other solutions as described in the following lemma. 
Lemma 3.8. If, in addition to the conditions of Lemma 3.6 and Lemma 3.7, $V^{0} \in L^{1}$ then there exists a positive constant $K_{8}$ such that the following is true for any $t>0$

$$
\left\|v_{1}(t)\right\|_{L^{\infty}} \leq K_{8}\left(\left\|V^{0}\right\|_{H^{1}}+\left\|V^{0}\right\|_{L^{1}}+\|W(0)\|_{H^{1}}\right) \frac{1}{t^{1 / 2}},
$$

Proof. The proof is similar to the proof of Lemma 3.7, but uses its results. We turn to (3.25) again,

$$
\begin{aligned}
v_{2}= & \mathrm{e}^{L_{2} t} v_{2}^{0} \\
& +\int_{0}^{t} \mathrm{e}^{L_{2}(t-s)}\left[\beta g_{1} w_{2}-\beta\left(g_{2}+g_{3}\right) w_{1}-\beta v_{1} v_{2} f_{1}+\dot{q}(s) h_{2}^{\prime}(\xi-q(s))\right] \mathrm{d} s .
\end{aligned}
$$

First we notice that instead of (3.27) we can in the same way obtain an estimate for $\left\|v_{2}(t)\right\|_{L^{1}}$. Indeed, because $g_{1}, g_{2}, g_{3}, h_{2}^{\prime}$ and $f_{1}$ are exponentially localized at $\pm \infty$, there are constants $\tilde{M}_{1}, \tilde{M}_{2}$ and $\tilde{M}_{3}, \tilde{M}_{7}$ and $\tilde{M}_{4}>0$ such that $\left\|g_{1}\right\|_{L^{1}} \leq \tilde{M}_{1}$, $\left\|g_{2}\right\|_{L^{1}} \leq \tilde{M}_{2},\left\|g_{3}\right\|_{L^{1}} \leq \tilde{M}_{3},\left\|h_{2}^{\prime}\right\|_{L^{1}} \leq \tilde{M}_{7},\left\|f_{1}\right\|_{L^{1}} \leq \tilde{M}_{4}$. We assume that initial perturbations are in $L^{1} \cap H^{1}$. The semigroup estimate (3.23) is valid in $L_{1}$ as well. Using the relation between norms, $\|\cdot\|_{L^{\infty}} \leq C\|\cdot\|_{H^{1}}$ for some $C>0$, from (3.25) we obtain

(3.40) $\left\|v_{2}(t)\right\|_{L^{1}}$

$$
\leq K_{4} \mathrm{e}^{-b t}\left\|v_{2}^{0}\right\|_{L^{1}}+C K_{4} \int_{0}^{t} \mathrm{e}^{-b(t-s)}\left(\beta\left(\tilde{M}_{1}+\tilde{M}_{2}+\tilde{M}_{3}\right) K_{2}+\tilde{M}_{7} K_{1}\right) \mathrm{e}^{-v s}\|W(0)\|_{H^{1}} \mathrm{~d} s,
$$

and in the same manner as in Lemma 3.6 we obtain the that there exists $\tilde{K}_{5}>0$ such that

$$
\left\|v_{2}(t)\right\|_{L^{1}} \leq \mathrm{e}^{-(v / 2) t} \tilde{K}_{5}\left(\left\|v_{2}^{0}\right\|_{L^{1}}+\|W(0)\|_{H^{1}}\right),
$$

for any $t<T_{\max }$. With (3.41) in mind, we turn to the equation for $v_{1}$.

The following semigroup estimates (3.35) for $\mathrm{e}^{L_{1} t}$ follow from the estimates on the heat kernel [4]: there exists a constant $K_{9}>0$ such that for any $z \in$ $L^{1} \cap H^{1} \subset L^{1} \cap L_{\infty}$

$$
\left\|\mathrm{e}^{L_{1} t} z(t)\right\|_{L^{\infty}} \leq K_{9} \min \left\{\frac{1}{t^{1 / 2}}\|z\|_{L^{1}},\|z\|_{L^{\infty}}\right\} .
$$


This estimate is used in the analysis of the variation-of-constant formula (3.36) rewritten for convenience as

$$
\begin{aligned}
& v_{1}=\mathrm{e}^{L_{1} t} v_{1}^{0} \\
& \qquad \begin{array}{l}
+\int_{0}^{t} \mathrm{e}^{L_{1}(t-s)}\left[\left(h_{2} \Omega_{h_{1}}\left(h_{1}(\xi-q(t))\right)+h_{2} f_{2}\right) \mathrm{e}^{-\alpha \xi} w_{1}+\dot{q}(t) h_{1}^{\prime}(\xi-q(t))\right] \mathrm{d} s \\
\quad+\int_{0}^{t} \mathrm{e}^{L_{1}(t-s)}\left(\Omega\left(h_{1}(\xi-q(t))\right) v_{2}+v_{1} f_{1} v_{2}\right) \mathrm{d} s .
\end{array}
\end{aligned}
$$

The functions $\left(h_{2} \Omega_{h_{1}}\left(h_{1}(\xi-q(t))\right)+h_{2} f_{2}\right) \mathrm{e}^{-\alpha \xi}$ and $h_{1}^{\prime}(\xi-q(t))$ are exponentially localized on $\pm \infty$, continuous functions, therefore are from $L_{1}$. Thanks to (3.14), (3.15) and (3.33) from Lemma 3.6, we then know that there exists a constant $K_{10}>0$ such that

$$
\begin{aligned}
\|\left(h_{2} \Omega_{h_{1}}\left(h_{1}(\xi-q(t))\right)+h_{2} f_{2}\right) \mathrm{e}^{-\alpha \xi} w_{1} & +\dot{q}(t) h_{1}^{\prime}(\xi-q(t)) \|_{L^{1}} \\
& \leq K_{10} \mathrm{e}^{-(v / 2) t}\left(\|W(0)\|_{H^{1}}+\left\|V^{0}\right\|_{H^{1}}\right),
\end{aligned}
$$

and, because of the relation between $L^{\infty}$ and $H^{1}$ norms, $K_{10}$ can be also chosen so

$$
\begin{aligned}
\|\left(h_{2} \Omega_{h_{1}}\left(h_{1}(\xi-q(t))\right)+h_{2} f_{2}\right) \mathrm{e}^{-\alpha \xi} w_{1} & +\dot{q}(t) h_{1}^{\prime}(\xi-q(t)) \|_{L^{\infty}} \\
& \leq K_{10} \mathrm{e}^{-(v / 2) t}\left(\|W(0)\|_{H^{1}}+\left\|V^{0}\right\|_{H^{1}}\right) .
\end{aligned}
$$

For the term with $v_{2}$ we use (3.41) and (3.33) from Lemma 3.6 to obtain

$$
\begin{gathered}
\left\|\Omega\left(h_{1}(\xi-q(t))\right) v_{2}\right\|_{L^{1}} \leq K_{11} \mathrm{e}^{-(v / 2) t}\left(\|W(0)\|_{H^{1}}+\left\|v_{2}^{0}\right\|_{L^{1}}\right), \\
\left\|\Omega\left(h_{1}(\xi-q(t))\right) v_{2}\right\|_{L^{\infty}} \leq K_{11} \mathrm{e}^{-(v / 2) t}\left(\|W(0)\|_{H^{1}}+\left\|v_{2}^{0}\right\|_{H^{1}}\right),
\end{gathered}
$$

Applying (3.33) again we then get

$$
\begin{aligned}
& \quad\left\|\int_{0}^{t} \mathrm{e}^{L_{1}(t-s)}\left(v_{1}(s) f_{1} v_{2}(s)\right) \mathrm{d} s\right\|_{L^{\infty}} \\
& \leq K_{9} \int_{0}^{t} \min \left\{\frac{1}{(t-s)^{1 / 2}}\left\|f_{1}\right\|_{L^{1}}\left\|v_{2}(s)\right\|_{H^{1}}\left\|v_{1}(s)\right\|_{L^{\infty}},\right. \\
& \left.\qquad f_{1}\left\|_{L^{\infty}}\right\| v_{2}(s)\left\|_{L^{\infty}}\right\| v_{1}(s) \|_{L^{\infty}}\right\} \mathrm{d} s \\
& \leq K_{12}\left(\|W(0)\|_{H^{1}}+\left\|V^{0}\right\|_{H^{1}}\right)\left(\|W(0)\|_{H^{1}}+\left\|v_{2}^{0}\right\|_{L^{1}}\right) \\
& \quad \times \int_{0}^{t} \min \left\{\frac{1}{(t-s)^{1 / 2}}, 1\right\} \mathrm{e}^{-(v / 2) s} \mathrm{~d} s,
\end{aligned}
$$


where $K_{12}=K_{9} K_{5} K_{7} \max \left\{M_{4}, C \tilde{M}_{4}\right\}$, and $C$ from $\left\|v_{2}\right\|_{L^{\infty}} \leq C\left\|v_{2}\right\|_{H^{1}}$. Therefore,

$$
\begin{aligned}
\left\|v_{1}(t)\right\|_{L^{\infty}} \leq & K_{9} \min \left\{\frac{1}{t^{1 / 2}}\left\|v_{1}^{0}\right\|_{L^{1}},\left\|v_{1}^{0}\right\|_{L^{\infty}}\right\} \\
& +K_{9}\left(K_{10}+K_{11}+K_{12}\left(\|W(0)\|_{H^{1}}\right.\right. \\
& \left.\left.\left\|v_{2}^{0}\right\|_{L^{1}}\right)\right)\left(\|W(0)\|_{H^{1}}+\left\|V^{0}\right\|_{H^{1}}\right) \\
& \times \int_{0}^{t} \min \left\{\frac{1}{(t-s)^{1 / 2}}, 1\right\} \mathrm{e}^{-\frac{v}{2} s} \mathrm{~d} s .
\end{aligned}
$$

We want to show that the right hand side of the previous inequality, when multiplied by $t^{1 / 2}$, is uniformly bounded in $t$. It is easy to see that

$$
\begin{aligned}
t^{1 / 2} \min \left\{\frac{1}{t^{1 / 2}}\left\|v_{1}^{0}\right\|_{L^{1}},\left\|v_{1}^{0}\right\|_{L^{\infty}}\right\} & \leq t^{1 / 2} \min \left\{\frac{1}{t^{1 / 2}}, 1\right\} \max \left\{\left\|v_{1}^{0}\right\|_{L^{1}},\left\|v_{1}^{0}\right\|_{L^{\infty}}\right\} \\
& \leq \max \left\{\left\|v_{1}^{0}\right\|_{L^{1}},\left\|v_{1}^{0}\right\|_{L^{\infty}}\right\} .
\end{aligned}
$$

There exists $K_{13}>0$ such that

$$
t^{1 / 2} \int_{0}^{t} \min \left\{\frac{1}{(t-s)^{1 / 2}}, 1\right\} \mathrm{e}^{-(v / 2) s} \mathrm{~d} s \leq K_{13}
$$

uniformly in $t$. Indeed,

$\int_{0}^{t} \min \left\{\frac{1}{(t-s)^{1 / 2}}, 1\right\} \mathrm{e}^{-(v / 2) s} \mathrm{~d} s=\int_{0}^{t-1} \frac{1}{(t-s)^{1 / 2}} \mathrm{e}^{-(v / 2) s} \mathrm{~d} s+\int_{t-1}^{t} \mathrm{e}^{-(v / 2) s} \mathrm{~d} s$.

The second integral is easily evaluated

$$
\int_{t-1}^{t} \mathrm{e}^{-(v / 2) s}=\frac{2}{v}\left(\mathrm{e}^{-(v / 2) t}-\mathrm{e}^{-(v / 2)(t-1)}\right) \leq \frac{2}{v}\left(1-e^{-1}\right) .
$$

To find a bound for the first integral, we make a substitution $z=t-s$,

$$
t^{1 / 2} \int_{0}^{t-1} \frac{1}{(t-s)^{1 / 2}} \mathrm{e}^{-(v / 2) s} \mathrm{~d} s=\frac{\int_{1}^{t} \frac{1}{z^{1 / 2}} \mathrm{e}^{(v / 2) z} \mathrm{~d} z}{t^{-1 / 2} \mathrm{e}^{(v / 2) t}} .
$$

L'Hôpital's rule shows that this function of $t$ at $+\infty$ approaches 1 , and therefore is bounded.

Finally, we obtain that

$$
\begin{aligned}
& t^{1 / 2}\left\|v_{1}\right\|_{L^{\infty}} \leq C K_{9} \max \left\{\left\|v_{1}^{0}\right\|_{L^{1}},\left\|v_{1}^{0}\right\|_{H^{1}}\right\} \\
& +C K_{9} K_{13}\left(K_{10}+K_{11}+K_{12}\left(\|W(0)\|_{H^{1}}+\left\|v_{2}^{0}\right\|_{L^{1}}\right)\right) \\
& \times\left(\|W(0)\|_{H^{1}}+\left\|V^{0}\right\|_{H^{1}}\right),
\end{aligned}
$$


from which (3.39) follows. The proof of the lemma is complete.

The choice of the weight restricts the class of admissible perturbations to those that decay exponentially fast at $+\infty$. In Lemma 3.8 we request that initial perturbations belong to $L^{1}$ space, but never need their $L^{1}$ norm to be small. In other words, we impose an additional condition only on the decay rates of perturbations at $-\infty$, but not on the size. The convergence is then slow, but it occurs in the norm without a weight.

Acknowledgments. A.G. expresses her deep gratitude to C. Jones and B. Sandstede for encouragement and useful discussions of this problem. A.G. wishes as well to thank Y. Latushkin and the anonymous referee for their comments on the details of the proof and on the paper in general. This work was supported by NSF grant DMS-0410267.

\section{REFERENCES}

[1] J. Alexander, R. Gardner, and C. Jones, A topological invariant arising in the stability analysis of travelling waves, J. Reine Angew. Math. 410 (1990), 167-212. MR 1068805 (92d:58028)

[2] S. Balasuriya, G. GotTwald, J. Hornibrook, and S. Lafortune, High Lewis number combustion wavefronts: a perturbative Melnikov analysis, SIAM J. Appl. Math. 67 (2007), 464486. MR 2285873

[3] H. Berestycki, B. NiCOLAENKO, and B. SCHEURER, Traveling wave solutions to combustion models and their singular limits, SIAM J. Math. Anal. 16 (1985), 1207-1242. MR 0807905 (87h:35326)

[4] T. Brand, M. Kunze, G. Schneider, and T. Seelbach, Hopf bifurcation and exchange of stability in diffusive media, Arch. Ration. Mech. Anal. 171 (2004), 263-296. MR 2034755(2005a:35154)

[5] N. FENICHEL, Geometric singular perturbation theory for ordinary differential equations, J. Differential Equations 31 (1979), 53-98. MR 0524817 (80m:58032)

[6] R. A. GARDNER and K. ZUMBRUN, The gap lemma and geometric criteria for instability of viscous shock profiles, Comm. Pure Appl. Math. 51 (1998), 797-855. MR 1617251 (99c:35152)

[7] A. GHAZARYAn, On the convective nature of the instability of a front undergoing a supercritical Turing instability, Math. Comput. Simulation (2008) (to appear).

[8] A. Ghazaryan, P. Gordon, and C. K. R. T. Jones, Traveling waves in porous media combustion: uniqueness of waves for small thermal diffusivity, J. Dynam. Differential Equations 19 (2007), 951-966. MR 2357533

[9] A. GHAZARYAN and C. K. R. T. JONES, On the stability of high Lewis number combustion fronts, Discrete Contin. Dynam. Systems-A 24-3 (to appear), Bates special issue.

[10] A. Ghazaryan, Y. Latushrin, S. Schecter, and A. De Souza, Stability of fronts in one-dimensional solid combustion (submitted for publication).

[11] A. Ghazaryan and B. SAndstede, Nonlinear convective instability of Turing-unstable fronts near onset: a case study, SIAM J. on Appl. Dyn. Syst. 6 (2007), 319-347. MR 2318657(2008d:37137) 
[12] P. GRINDROD, Patterns and waves: The theory and applications of reaction-diffusion equations, Oxford Applied Mathematics and Computing Science Series, The Clarendon Press, Oxford University Press, New York, 1991. MR 1136256 (92k:35145)

[13] V. Gubernov, G. N. Mercer, H. S. Sidhu, and R. O. Weber, Evans function stability of combustion waves, SIAM J. Appl. Math. 63 (2003), 1259-1275. MR 1989902 (2004e:35118)

[14] D. Henry, Geometric theory of semilinear parabolic equations, Springer Lecture Notes in Mathematics, vol. 840, Springer-Verlag, Berlin-New York, 1981, ISBN. MR 0610244 (83j:35084)

[15] C. K. R. T. JONES, R. GARDNER, and T. KAPITULA, Stability of travelling waves for nonconvex scalar viscous conservation laws, Comm. Pure Appl. Math. 46 (1993), 505-526. MR 1211740 (94c:35123)

[16] C. K. R. T. Jones, Geometric singular perturbation theory, Dynamical Systems 1609 (1995), 44-118. MR 1374108 (97e:34105)

[17] J. I. KANEL', On steady state solutions to systems of equations arising in combustion theory, Dokl. Akad. Nauk USSR 149 (1963), 367-369.

[18] T. KAPITULA, On the stability of travelling waves in weighted $L^{\infty}$ spaces, J. Differential Equations 112 (1994), 179-215. MR 1287557 (95h:35107)

[19] G. Lyng, M. Raoofi, B. Texier, and K. Zumbrun, Pointwise Green function bounds and stability of combustion waves, J. Differential Equations 233 (2007), 654-698. MR 2292522 (2007m:35147)

[20] A. PAZY, Semigroups of linear operators and applications to partial differential equations, Applied Mathematical Sciences, vol. 44, Springer-Verlag, New York, 1983. MR 0710486 (85g:47061)

[21] R. L. Pego and M. I. Weinstein, Asymptotic stability of solitary waves, Comm. Math. Phys. 164 (1994), 305-349. MR 1289328 (95h:35209)

[22] B. SANDSTEDE and A. SCHEEL, Absolute and convective instabilities of waves on unbounded and large bounded domains, Phys. D 145 (2000), 233-277. MR 1782392 (2002k:37157)

[23] B. SAndstede, Stability of travelling waves, Handbook of Dynamical Systems 2 (2002), 9831055. MR 1901069 (2004e:37121)

[24] D. H. SATtinger, On the stability of waves of nonlinear parabolic systems, Advances in Math. 22 (1976), 312-355. MR 0435602 (55 \#8561)

[25] P. Simon, J. H. MERKIN, and S. K. SCOTT, Bifurcations in non-adiabatic flame propagation models, Focus on Combustion Research (2006), 315-357.

[26] F. VARAS and J. M. VEGA, Linear stability of a plane front in solid combustion at large heat of reactions, SIAM J. Appl. Math. 62 (2002), 1810-1822. MR 1918578 (2003f:35172)

[27] Y. WU, The stability of travelling fronts for some quasilinear Burgers-type equations, Adv. Math. (China) 31 (2002), 363-371. MR 1930403 (2003g:35147)

[28] Y. WU and X. XING, The stability of travelling fronts for general scalar viscous balance law, J. Math. Anal. Appl. 305 (2005), 698-711. MR 2131532 (2006b:35221)

[29] K. ZUMBRUN and P. HOWARD, Pointwise semigroup methods and stability of viscous shock waves, Indiana Univ. Math. J. 47 (1998), 741-871. MR 1665788 (99m:35157)

[30] K. ZUMBRUN and D. SERRE, Viscous and inviscid stability of multidimensional planar shock fronts, Indiana Univ. Math. J. 48 (1999), 937-992. MR 1736972 (2001h:35122) 
University of North Carolina

Chapel Hill, NC, U.S.A.

E-MAIL: ghazarya@email.unc.edu

KEY WORDS AND PHRASES: traveling wave, nonlinear stability, exponential weights, high Lewis number, combustion front.

2000 Mathematics SubJECt Classification: 35B35,35K57, 80A25.

Received: November 29th, 2007; revised: April 3rd, 2008. 